\title{
The SAMI Galaxy Survey: Spatially resolved metallicity and ionization mapping
}

Henry Poetrodjojo, ${ }^{1,2 \star}$ Brent Groves, ${ }^{1,2}$ Lisa J. Kewley, ${ }^{1,2}$ Anne M. Medling, ${ }^{1,3} \dagger$ Sarah M. Sweet, ${ }^{2,4}$ Jesse van de Sande ${ }^{5}$ Sebastian F. Sanchez, ${ }^{6}$ Joss Bland-Hawthorn, ${ }^{5}$ Sarah Brough, ${ }^{7}$ Julia J. Bryant, ${ }^{5,8,9}$ Luca Cortese, ${ }^{2,10}$ Scott M. Croom, ${ }^{5,9}$ Ángel R. López-Sánchez, ${ }^{2,8,11}$ Samuel N. Richards, ${ }^{12}$ Tayyaba Zafar, ${ }^{8}$ Jon S. Lawrence, ${ }^{8}$ Nuria P. F. Lorente, ${ }^{8}$ Matt S. Owers ${ }^{8,11}$ and Nicholas Scott ${ }^{5}$

${ }^{1}$ Research School of Astronomy and Astrophysics, The Australian National University, Canberra, ACT 2611, Australia

${ }^{2} A R C$ Centre of Excellence for All Sky Astrophysics in 3 Dimensions (ASTRO 3D)

${ }^{3}$ Cahill Center for Astronomy and Astrophysics, California Institute of Technology, MS 249-17 Pasadena, CA 91125, USA

${ }^{4}$ Centre for Astrophysics and Supercomputing, Swinburne University of Technology, PO Box 218, Hawthorn, VIC 3122, Australia

${ }^{5}$ Sydney Institute for Astronomy (SIfA), School of Physics, University of Sydney, Sydney, NSW 2006, Australia

${ }^{6}$ Instituto de Astronomía, Universidad Nacional Autónoma de México, A.P. 70-264, 04510 Mexico city, Mexico

${ }^{7}$ School of Physics, University of New South Wales, Kensington, NSW 2052, Australia

${ }^{8}$ Australian Astronomical Observatory, 105 Delhi Rd, North Ryde, NSW 2113, Australia

${ }^{9}$ ARC Centre of Excellence for All-sky Astrophysics (CAASTRO)

${ }^{10}$ International Centre for Radio Astronomy Research, University of Western Australia, 35 Stirling Highway, Crawley, WA 6009, Australia

${ }^{11}$ Department of Physics and Astronomy, Macquarie University, North Ryde, NSW 2109, Australia

${ }^{12}$ SOFIA Operations Center, USRA, NASA Armstrong Flight Research Center, 2825 East Avenue P, Palmdale, CA 93550, USA

Accepted 2018 July 3. Received 2018 July 1; in original form 2018 April 5

\begin{abstract}
We present gas-phase metallicity and ionization parameter maps of 25 star-forming face-on spiral galaxies from the SAMI Galaxy Survey Data Release 1. Self-consistent metallicity and ionization parameter maps are calculated simultaneously through an iterative process to account for the interdependence of the strong emission line diagnostics involving $\left(\left[\mathrm{O}_{\mathrm{II}}\right]+[\mathrm{O} \mathrm{III}]\right) / \mathrm{H} \beta$ $\left(\mathrm{R}_{23}\right)$ and $\left[\mathrm{O}_{\mathrm{III}}\right] /\left[\mathrm{O}_{\mathrm{II}}\right](\mathrm{O} 32)$. The maps are created on a spaxel-by-spaxel basis because $\mathrm{H}_{\mathrm{II}}$ regions are not resolved at the SAMI spatial resolution. We combine the SAMI data with stellar mass, star formation rate (SFR), effective radius $\left(R_{\mathrm{e}}\right)$, ellipticity, and position angles (PA) from the GAMA survey to analyse their relation to the metallicity and ionization parameter. We find a weak trend of steepening metallicity gradient with galaxy stellar mass, with values ranging from -0.03 to $-0.20 \mathrm{dex} / R_{\mathrm{e}}$. Only two galaxies show radial gradients in the ionization parameter. We find that the ionization parameter has no significant correlation with either SFR, sSFR (specific SFR), or metallicity. For several individual galaxies, we find the structure in the ionization parameter maps suggestive of spiral arm features. We find a typical ionization parameter range of $7.0<\log (q)<7.8$ for our galaxy sample with no significant overall structure. An ionization parameter range of this magnitude is large enough to caution the use of metallicity diagnostics that have not considered the effects of a varying ionization parameter distribution.
\end{abstract}

Key words: galaxies: abundances - galaxies: ISM.

\section{INTRODUCTION}

The accurate measurement of gas-phase metallicity and ionization parameter in galaxies is becoming increasingly essential as we probe deeper into the Universe and observe galaxies at high redshift. The gas-phase metallicity is strongly affected by processes that occur during the evolution of galaxies such as gas inflows, galaxy mergers, and galactic winds. Because of this connection, the distribution of the metallicity in galaxies provides a strong constraint on their growth and formation and recent dynamical processes.

${ }^{\star}$ E-mail: henry.poetrodjojo@anu.edu.au
$\dagger$ Hubble fellow 
Simulations by Pilkington et al. (2012) show that a negative gasphase metallicity gradient provides strong evidence for inside-out disc formation (Matteucci \& Francois 1989; Boissier \& Prantzos 1999). In this model, a negative metallicity gradient implies that the central metal-rich gas has been forming stars for longer than the metal-poor outskirts.

Local disc galaxies typically have a negative metallicity gradient (Zaritsky, Kennicutt \& Huchra 1994; Moustakas et al. 2010; Rupke, Kewley \& Chien 2010; Sánchez et al. 2014). A dependence on morphology was observed by Vila-Costas \& Edmunds (1992), Zaritsky et al. 1994, and Martin \& Roy 1994, in that barred galaxies have shallower metallicity gradients than unbarred galaxies.

Large-scale gas inflows can disrupt metallicity gradients. Kewley et al. (2010) showed that the metallicity gradients of close pair galaxies are significantly shallower than those of isolated galaxies. Tidal disruptions from galaxy interactions drive pristine gas from the outskirts into the central regions, diluting the metal-rich centre. López-Sánchez et al. (2015) showed that one of the spiral arms belonging to NGC 1512 had a flattened metallicity gradient due to its interaction with nearby dwarf galaxy NGC 1510. Sánchez et al. (2014) also found significantly flatter metallicity gradients in galaxies that show signs of merger activity.

With advances in integral field spectroscopy (IFS), we can now spatially map the metallicity across galaxies, allowing for a deeper insight into azimuthal and radial variations within a galaxy. Several small-scale surveys such as PPAK IFS Nearby Galaxies Survey (PINGS; Rosales-Ortega et al. 2010), the VIRUS-P Investigation of the Extreme Environments of Starbursts (VIXENS; Heiderman et al. 2011), and the VIRUS-P Exploration of Nearby Galaxies (VENGA; Blanc et al. 2013; Kaplan et al. 2016) have been conducted. The first large survey was the Spectrographic Areal Unit for Research on Optical Nebulae (SAURON) survey (de Zeeuw et al. 2002), which initially observed 72 low-redshift early-type galaxies using IFS technology, and was later continued into the ATLAS ${ }^{3 \mathrm{D}}$ survey (Cappellari et al. 2011), observing 260 galaxies at $z<0.01$.

The Calar Alto Legacy Integral Field Area Survey (CALIFA) survey (Sánchez et al. 2012) consists of 600 galaxies with $z<$ 0.03. Sánchez et al. (2014) used 306 CALIFA galaxies to analyse the oxygen abundance gradients in galaxy discs and found that all undisturbed galaxies with a disc presented similar radial metallicity gradients when normalized to the size of the disc. They showed that the existence of a characteristic metallicity gradient is independent of luminosity, mass, and morphology when normalized to the size of the disc. Similar results were obtained by Sánchez et al. (2012) using PINGS data, and Ho et al. (2015) and Sánchez-Menguiano et al. (2016a) who both used CALIFA data for their analysis. This contradicts the findings of Vila-Costas \& Edmunds (1992), Zaritsky et al. 1994, and Martin \& Roy 1994 who found a clear variation in metallicity gradient between barred and unbarred galaxies. These differences could be due to earlier studies using a smaller sample size (Ho et al. 2015) or inconsistent methods of measuring metallicity gradients (Sánchez-Menguiano et al. 2016a).

While the ATLAS ${ }^{3 \mathrm{D}}$ and the CALIFA surveys have now managed to amass hundreds of galaxies, they do not have the multiplexing technology to easily reach thousands of galaxies. This was made possible by the development of the hexabundle (BlandHawthorn et al. 2011), which led to the development of the SydneyAAO Multi-object Integral field (SAMI) spectrograph (Croom et al. 2015). The SAMI Galaxy Survey (Bryant et al. 2015) will complete in 2018 with 3600 galaxies across a wide range of environments and stellar masses, allowing for the disentanglement of degeneracies. This will be followed by the Hector survey with an order of magni- tude increase in the observed number of galaxies (Bland-Hawthorn 2015).

The Mapping Nearby Galaxies at Apache Point Observatory (MaNGA) survey (Bundy et al. 2015) is an ongoing galaxy survey aiming at achieving spatially resolved spectra of 10000 nearby galaxies. MaNGA uses specially designed fibre bundles (Drory et al. 2015) that vary in diameter and number of fibres to allow the observation of a representative sample of local galaxies in the mass range $10^{9}<M / \mathrm{M}_{\odot}<10^{12}$. Fibre bundles range from 19 to 127 fibres with an on-sky diameter ranging from $12 \operatorname{arcsec}$ to $32 \mathrm{arcsec}$. With a sample of 550 galaxies from the MaNGA survey, Belfiore et al. (2017) found a steepening of the metallicity gradients with stellar mass up to a mass of $\log \left(M_{*} / \mathrm{M}_{\odot}\right)<10.5$. For more-massive galaxies, the metallicity gradient flattens slightly as the metallicity of the galaxy reaches a constant value.

The gas-phase metallicity is most commonly presented as the ratio between the abundance of oxygen, the most-abundant heavy element by mass, and hydrogen. For star-forming galaxies, the metallicity is usually determined using the ratios of the strong emission lines. Some of the popular strong emission line diagnostics include $\left(\left[\mathrm{O} \sim_{\mathrm{II}}\right] \lambda 3726, \lambda 3729+\left[\mathrm{O} \sim_{\mathrm{III}}\right] \lambda 4959, \lambda 5007\right) / \mathrm{H} \beta$ $\left(\mathrm{R}_{23}\right.$; Kobulnicky \& Kewley 2004, hereafter KK04), $\left[\mathrm{N} \sim_{\mathrm{II}}\right] \lambda 6583 /\left[\mathrm{O} \sim_{\mathrm{II}}\right] \lambda 3726, \lambda 3729(\mathrm{~N} 2 \mathrm{O} 2$; Kewley \& Dopita 2002, hereafter KD02), ([O $\left.\left.\sim_{\mathrm{III}}\right] \lambda 5007 / \mathrm{H} \beta\right) /\left(\left[\mathrm{N} \sim_{\mathrm{II}}\right] \lambda 6583 / \mathrm{H} \alpha\right)(\mathrm{O} 3 \mathrm{~N} 2$; Pettini \& Pagel 2004, hereafter PP04), [N $\left.\sim_{I I}\right] \lambda 6583 / \mathrm{H} \alpha$ (N2HA;

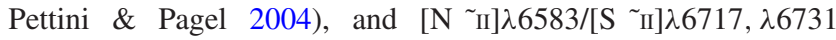
(N2S2; Dopita et al. 2016, hereafter D16). Each diagnostic has its own set of advantages and disadvantages making them suitable for different situations. These diagnostics are then calibrated against data to determine metallicities. However, all these metallicity calibrations are inconsistent with each other, leading to different abundances, depending on the particular diagnostic and calibration used. Kewley \& Ellison (2008) attempt to consolidate the many metallicity diagnostics and calibrations by providing conversion polynomials between them. For a comprehensive review and analysis of the various metallicity diagnostics and calibrations, see Kewley \& Ellison (2008) and López-Sánchez et al. (2012).

Ionization parameter strongly affects many metallicity diagnostics (e.g. N2HA, O3N2, and $\mathrm{R}_{23}$ ). The ionization parameter is defined as follows:

$q=\frac{S_{H^{0}}}{n}$

where $S_{H^{0}}$ is the ionizing photon flux per unit area and $n$ is the number density of the interstellar medium (ISM). The ionization parameter is a measure of the amount of ionizing photons passing through the ISM per hydrogen atom. Dopita et al. (2014) found a strong correlation between ionization parameter and star formation rate (SFR) and suggest that the correlation is caused by the change in geometry of the molecular and ionized gas with environment. Similar results are obtained by Kaplan et al. (2016), who found strong evidence of the existence of radial ionization parameter gradients and a correlation with SFR.

The dependence of metallicity diagnostics on the ionization parameter is clearly shown in López-Sánchez et al. (2011) and Ho et al. (2015). The KD02 N2O2 diagnostic is relatively independent of ionization parameter, but the PP04 O3N2 diagnostic was empirically calibrated without taking into account the effect of the ionization parameter. Ho et al. (2015) showed that the differences between the two diagnostics correlate strongly with the ionization parameter, highlighting the importance of correcting for ionization parameter when calculating metallicity. 
In this paper, we simultaneously constrain the metallicity and ionization parameter of pure star-forming SAMI galaxies through an iterative process and produce self-consistent spatially resolved metallicity and ionization parameter maps. We derive metallicity gradients and analyse the spatial distribution of the ionization parameter. We confirm the results of Sánchez et al. (2012, 2014), Ho et al. (2015), and Sánchez-Menguiano et al. (2016a) by obtaining consistent metallicity gradient values. We find a weak massdependence of metallicity gradients using the KK04 R 23 metallicity diagnostic, showing a similar trend to Belfiore et al. (2017). We show that the ionization parameter does not change as a function of radius with most star-forming galaxies, and we investigate whether the ionization parameter correlates with fundamental galaxy properties like metallicity, SFR, and specific SFR (sSFR). Finally, we show the implications of excluding the ionization parameter from metallicity calculations. We structure this paper in the following way. Section 2 describes the SAMI Galaxy Survey and how we select our sub-sample from the data available. We outline the methods we use for determining the metallicity and ionization parameter while taking into account the interdependence of the diagnostics in Section 3. In Sections 4 and 5, we present and briefly compare to previous work our results of the metallicity and ionization parameter analysis, respectively. We discuss the results and provide a summary and conclusion in Sections 6 and 7, respectively. Throughout the entire paper, we assume the following values for cosmological constants, $H_{0}=70 \mathrm{~km} \sim^{-1} \mathrm{Mpc}^{-1}, \Omega_{\mathrm{M}}=0.3$, and $\Omega_{\Lambda}=0.7$.

\section{SAMPLE SELECTION}

\subsection{SAMI Galaxy Survey}

The SAMI Galaxy Survey (Croom et al. 2012) is an ongoing integral field spectroscopic survey of $\sim 3600$ low-redshift $(z<\$ 0.12)$ galaxies primarily selected from the Galaxy and Mass Assembly (GAMA) survey (Driver et al. 2011), with the addition of eight galaxy clusters to extend the sampling of environmental density (Owers et al. 2017). The survey uses the SAMI spectrograph on the 3.9 metre Anglo-Australian Telescope at Siding Spring Observatory. The final primary survey targets consist of galaxies with stellar masses between $10^{7}$ and $10^{12} \mathrm{M}_{\odot}$, redshifts between 0.004 $<z<0.095$, and magnitudes $r_{\text {pet }}<19.4$ mag. For full details on the SAMI Galaxy Survey selection, refer to Bryant et al. (2015). The SAMI data are released as a red and blue data cubes for each galaxy, with $50 \times 500.25(0.5 \times 0.5) \operatorname{arcsec}^{2}$ spaxels covering the 14.7 arcsec diameter aperture of the SAMI hexabundle and an average seeing of 2.16 arcsec (see Green et al. 2017 for details). The blue cube covers a wavelength range between 3700 and $5700 \AA$; with a spectral resolution of $R=1812$ and the red cube covers a wavelength range between 6300 and $7400 \AA$; with a spectral resolution of $R=4263$ (van de Sande et al. 2017). These spectral ranges cover the strong optical emission lines commonly used as diagnostics of the gas-phase metallicity and ionization parameter: [O II] $\lambda 3726, \lambda 3729, \mathrm{H} \beta \lambda 4861,[\mathrm{O}$ III] $\lambda 5007, \mathrm{H} \alpha \lambda 6563,[\mathrm{~N}$ II] $\lambda 6583$ and $\left[\mathrm{S}_{\mathrm{II}}\right] \lambda 6717, \lambda 6731$. The red and blue data cubes are analysed using LaZy-IFU (LZIFU v0.3.2; Ho et al. 2016). LZIFU extracts total line fluxes for the dominant emission lines by fitting and subtracting the underlying continuum and then fitting the dominant emission lines using up to three Gaussian profiles. LZIFU returns maps of the flux and flux errors for each emission line, as well as maps of the ionized gas velocity and velocity dispersion and their associated errors (see Ho et al. 2016 for a detailed explanation of the routine).
The galaxy sample for which we determine the resolved metallicity and ionization parameter is based on the 772 galaxies in Data Release 1 of the SAMI Galaxy Survey (Green et al. 2017). However, to obtain the highest S/N (Signal-to-Noise) and largest possible maps of these parameters, we placed the following selection criteria on the galaxies (each of which is elaborated in the following subsections):

(i) Star-forming galaxies free of active galactic nuclei (AGN) and shocks using the Kewley et al. (2006) classification scheme.

(ii) Emission line maps covering at least 70 percent of the hexabundle field of view in all emission lines used.

(iii) Face-on galaxies with an inclination angle less than 60 degrees based on measurements from the GAMA survey.

(iv) Each galaxy is sampled to at least 1 effective radius $\left(R_{\mathrm{e}}<\right.$ $7.4 \mathrm{arcsec}$ ) based on measurement from the GAMA survey.

(v) A S/N ratio $>3$ in the [O II], $\mathrm{H} \beta$, [O III], $\mathrm{H} \alpha$, [N II], and [S II] emission line fluxes for each spaxel.

These selection criteria limit our sample to 25 star-forming, 'bestcase' scenario galaxies to determine reliable metallicity and ionization parameter maps. The final sample of galaxies and their global properties as defined in the GAMA galaxy catalogue are given in Table 1 . We use the $R$-band effective radii throughout this study. We also give the $\mathrm{H} \alpha$-derived SFR assuming a Salpeter (Salpeter 1955 ) initial mass function as well as stellar mass derived from the mass-luminosity relation (Taylor et al. 2011). For a comparison between the SFR values determined with GAMA data and SAMI data, see Medling et al. (2018). In future studies, we intend to expand this analysis to the full SAMI Galaxy Survey sample. With a larger sample, we will probe the relationships between metallicity and ionization parameter with galaxy properties in greater detail.

\subsection{Star-forming Galaxies}

For typical blue cloud galaxies, strong emission lines arise predominantly from $\mathrm{H}$ in regions surrounding recently formed massive stars. However, emission lines can also arise from gas excited by other sources of ionization, such as shocks or AGN, e.g. Groves, Dopita \& Sutherland (2004). The metallicity and ionization parameter diagnostics that we rely on are calibrated assuming $\mathrm{H}$ II region emission and cannot be simply applied to galaxies with significant contribution from other ionizing sources to the emission lines. In some cases, it is possible to separate the star-formation-dominated and other ionizing-sourced line emission (e.g. Davies et al. 2014, 2016), but in our case, we chose to remove all galaxies that showed significant non-star-forming emission. Medling et al. (2018) created star formation masks for the SAMI Galaxy Survey DR1 using the classification scheme of Kewley et al. (2006), which uses strong emission line ratios to create diagnostic curves that distinguish when non-star-forming emission is present:

$$
\begin{aligned}
& \log \frac{[\mathrm{O} \sim \mathrm{III}]}{\mathrm{H} \beta}>\frac{0.61}{\log \frac{\left[\mathrm{N}^{\sim} \mathrm{II}\right]}{\mathrm{H} \alpha}-0.05}+1.30, \\
& \log \frac{\left[\mathrm{O} \tilde{\mathrm{III}}^{\mathrm{II}}\right]}{\mathrm{H} \beta}>\frac{0.72}{\log \frac{\left[\mathrm{S}_{\mathrm{II}}\right]}{\mathrm{H} \alpha}-0.32}+1.30, \\
& \log \frac{[\mathrm{O} \sim \mathrm{III}]}{\mathrm{H} \beta}>\frac{0.73}{\log \frac{\left[\mathrm{O}_{\mathrm{I}}\right]}{\mathrm{H} \alpha}+0.59}+1.33 .
\end{aligned}
$$

Spaxels with a $\mathrm{S} / \mathrm{N}>5$ in the emission line fluxes that satisfy any (fail all) of these criteria were classified as non-star-forming 
Table 1. Selected sample of galaxies from the SAMI Galaxy Survey and their properties used for our analysis, obtained from the GAMA survey. SFR was not available for GAMA-78667 and GAMA-144402.

\begin{tabular}{|c|c|c|c|c|c|c|c|c|}
\hline GAMA & $\begin{array}{l}\text { RA } \\
\text { (deg) }\end{array}$ & $\begin{array}{l}\text { Dec } \\
\text { (deg) }\end{array}$ & Redshift & $\begin{array}{l}\log \text { (mass) } \\
\left(M_{*} / \mathrm{M}_{\odot}\right)\end{array}$ & $\begin{array}{c}\mathrm{SFR} \\
\left(\mathrm{M}_{\odot} \mathrm{yr}^{-1}\right)\end{array}$ & $\begin{array}{c}R_{\mathrm{e}} \\
(\operatorname{arcsec})\end{array}$ & $\begin{array}{l}\text { Ellipticity } \\
(1-(b / a))\end{array}$ & PA \\
\hline 008353 & 182.0164 & 0.6976 & 0.020 & 9.35 & 0.51 & 5.37 & 0.373 & 58.9 \\
\hline 030890 & 177.2579 & -1.1025 & 0.020 & 9.79 & 0.76 & 7.56 & 0.435 & 27.1 \\
\hline 053977 & 176.0183 & -0.2109 & 0.048 & 9.94 & 5.02 & 3.79 & 0.202 & 103.7 \\
\hline 077754 & 214.6477 & 0.1577 & 0.053 & 10.47 & 9.19 & 7.03 & 0.438 & 81.2 \\
\hline 078667 & 218.0908 & 0.1781 & 0.055 & 10.14 & - & 6.85 & 0.225 & 19.7 \\
\hline 100192 & 185.9276 & 0.9621 & 0.024 & 9.33 & 0.18 & 5.66 & 0.080 & 127.3 \\
\hline 106717 & 217.0188 & 1.0063 & 0.026 & 10.16 & 3.25 & 5.23 & 0.145 & 153.6 \\
\hline 144402 & 179.9611 & -1.3819 & 0.036 & 10.25 & - & 4.14 & 0.296 & 23.4 \\
\hline 184415 & 176.3419 & -1.5652 & 0.028 & 9.54 & 0.50 & 3.62 & 0.352 & 134.7 \\
\hline 209181 & 132.1251 & 0.1708 & 0.058 & 10.24 & 3.71 & 4.31 & 0.442 & 120.6 \\
\hline 209743 & 134.6767 & 0.1914 & 0.041 & 10.16 & 2.15 & 6.95 & 0.137 & 10.1 \\
\hline 422366 & 130.5955 & 2.4973 & 0.029 & 9.64 & 0.41 & 8.86 & 0.354 & 168.7 \\
\hline 463288 & 212.4848 & -1.2400 & 0.025 & 9.63 & 2.48 & 7.26 & 0.183 & 121.6 \\
\hline 487027 & 222.6791 & -1.7148 & 0.026 & 10.11 & 9.04 & 6.22 & 0.408 & 31.6 \\
\hline 492414 & 216.5031 & -1.4117 & 0.055 & 10.06 & 1.39 & 4.40 & 0.240 & 110.9 \\
\hline 610997 & 182.8690 & 0.3786 & 0.020 & 9.32 & 0.21 & 5.48 & 0.043 & 179.6 \\
\hline 618116 & 214.4055 & 0.3290 & 0.051 & 10.24 & 2.16 & 5.76 & 0.181 & 166.5 \\
\hline 622744 & 134.8299 & 0.7977 & 0.013 & 9.07 & 1.42 & 5.39 & 0.227 & 52.4 \\
\hline
\end{tabular}

(star-forming). In the case where $\mathrm{S} / \mathrm{N}<5$, Medling et al. (2018) used a conservative approach to ensure that the sample remained clean.

After identifying the dominant ionization mechanism in each spaxel, Medling et al. (2018) calculated the fraction of the hexabundle field of view that is filled by the star-forming spaxels. For our analysis, we require that 70 per cent of the hexabundle was star forming to ensure that a significant portion of the field of view is filled. This reduces our DR1 SAMI galaxy sample to 91 galaxies. Implementing this sample selection cut excludes galaxies based on several other galaxy properties. This cut clearly removes galaxies belonging to the red sequence, leaving only galaxies that lie within the blue cloud. However, we are also performing cuts based on angular size and ellipticity. Since we require at least 70 per cent of the hexabundle to be filled with star-forming spaxels, we remove both small blue galaxies as well as highly inclined galaxies that do not sufficiently fill the field of view. 92 galaxies have star formation fractions (fraction of $\mathrm{H} \alpha$ spaxels classified as star forming) less than 10 percent. This subset is filled with red sequence galaxies that no longer undergo significant star formation. Of the remaining 680 blue cloud galaxies, 151 (22 per cent) galaxies have star formation fractions greater than 70 per cent. For 91 (13 per cent) galaxies, the star-forming spaxels also fill 70 per cent of the total hexabundle field of view. Although 60 galaxies have star formation fractions greater than 70 per cent, their angular size is either too small or are too inclined to fill the hexabundle field of view. Overall, this cut removes non-star-forming elliptical galaxies as well as AGN and shock-dominated galaxies, where the majority of spaxels satisfy the diagnostic curves shown in equations (2)-(4). Although small low surface brightness galaxies have high star formation fractions with respect to their size, their angular size is not large enough to sufficiently fill the hexabundle, making it difficult to derive radial gradients.

\subsection{Well-resolved radial profiles}

To measure reliable radial metallicity gradients, we require wellsampled radial profiles of the emission line fluxes. In practice, this means that we select galaxies with inclinations of $<60^{\circ}$ and effective radii $R_{\mathrm{e}}<7.4$ arcsec for face-on galaxies to ensure that we sample at least five resolution elements across $1 R_{\mathrm{e}}$ and that we limit confusion along the minor axis. These selection criteria further reduce our galaxy sample to 38 galaxies.

\subsection{High S/N Galaxies}

To obtain reliable metallicity and ionization parameter measurements, we require spaxels to have a $\mathrm{S} / \mathrm{N}>3$ in all of the emission line fluxes used in our diagnostic ratios: [O II] $\lambda 3726, \lambda 3729, \mathrm{H} \beta \lambda 4861$, $[\mathrm{O}$ III] $] 25007, \mathrm{H} \alpha \lambda 6563,\left[\mathrm{~N}_{\text {II }}\right] \lambda 6583$, and $\left[\mathrm{S}_{\text {II }}\right] \lambda 6717, \lambda 6731$. We applied this criterion to all spaxels in our remaining galaxy sample, while still requiring a coverage of 70 per cent of the SAMI field of view. This final cut, especially the limit on [O II], reduced our sample to 28 galaxies. A further three galaxies had such a small redshift such that the [O II] emission line was not redshifted enough to fall in the range of the detector. Applying this final cut reduces the final sample to 25 face-on resolved star-forming galaxies. Fig. 1 compares our sample to all the galaxies in DR1 of the SAMI Galaxy Survey. It is clear that our sample is extremely biased with respect to the SAMI Galaxy Survey. The low-mass galaxies have an effective radii distribution similar to the whole DR1 sample. Since these lowmass galaxies are spread over the same area as higher mass galaxies, they are more diffuse and hence harder to detect to a reliable $\mathrm{S} / \mathrm{N}$. 


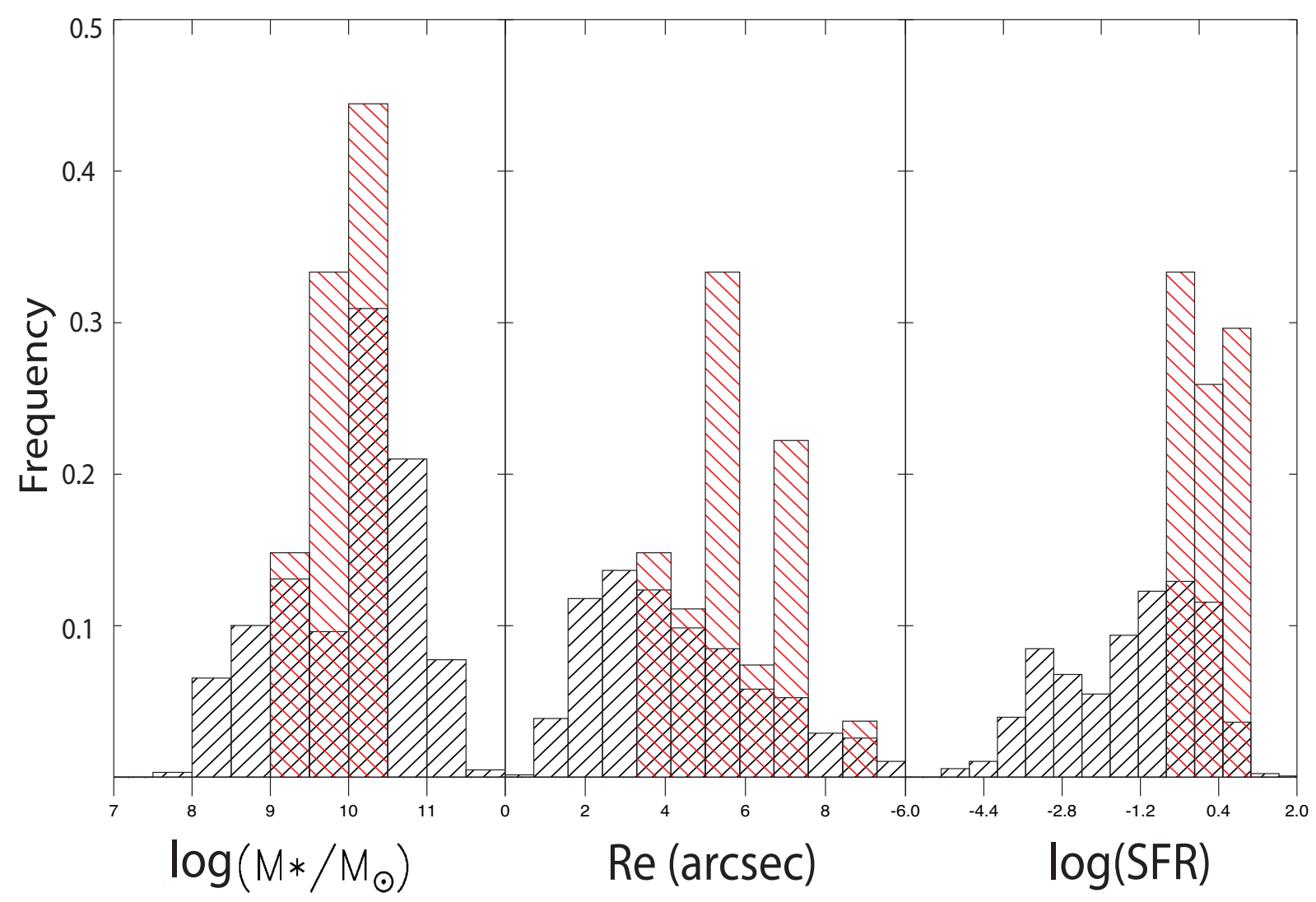

Figure 1. Comparison between DR1 of the SAMI Galaxy Survey (black) and the final galaxy sample used for our analysis (red). Left-hand panel: We have selected galaxies in the middle of the mass range $\left(9.0<\log M_{*} / \mathrm{M}_{\odot}<10.5\right)$ of DR1. Less-massive galaxies are selected to have smaller redshifts and have comparable effective radii. This means that they are more diffuse and harder to obtain reliable $\mathrm{S} / \mathrm{N}$, leading to the lower mass limit. The upper mass limit is due to our restriction on sampling to at least $1 R_{\mathrm{e}}$. As we are observing a fairly narrow redshift range, more-massive galaxies tend to have a larger apparent size, meaning we are unable to achieve the minimum $1 R_{\mathrm{e}}$ we desire. Middle panel: Again, we sample the middle range of effective radii for the same reasons as we sample the middle range of stellar mass. The only exception is a slight spike beyond $R_{\mathrm{e}}>8$ arcsec. This comes from GAMA-422366, which has an ellipticity of 0.354 , allowing it to be sampled beyond $1 R_{\mathrm{e}}$ along the minor axis despite the effective radius being larger than the SAMI field of view radius. Right-hand panel: Since we are aiming at only looking at galaxies with high SFR fractions, we are only sampling the high-SFR end of the DR1 SAMI Galaxy Survey.

The $\mathrm{S} / \mathrm{N}$ requirements outlined in Medling et al. (2018) mean that low $\mathrm{S} / \mathrm{N}$ spaxels are usually classified as non-star-forming, causing the lower mass limit. The upper limit of about $\log \left(M_{*} / \mathrm{M}_{\odot}\right)=10.5$ is due to the fact that the blue sequence turns over at $\log \left(M_{*} / \mathrm{M}_{\odot}\right)$ $\approx 10.5$ (Karim et al. 2011), with more massive galaxies belonging to the non-star-forming red sequence. We sample the middle range of effective radii due to our requirements on sampling to at least $1 R_{\mathrm{e}}$ and filling 70 per cent of the hexabundle. We would not be able to sufficiently sample large angular size galaxies out to $1 R_{\mathrm{e}}$, and small angular size galaxies would not cover enough of the hexabundle. We have purposely selected galaxies to have high SFR, leading to the extreme bias towards high-SFR galaxies compared to the DR1 sample. Since smaller galaxies tend to be the one with low SFR, by removing all the low-mass galaxies, we are left only with very high star-forming galaxies.

\section{DETERMINING METALLICITY AND IONIZATION PARAMETER}

\subsection{Extinction correction}

We first correct the emission lines for the attenuation by dust in the ISM. The attenuation of emission lines is wavelength dependent, meaning that emission line diagnostics that use emission lines with wide wavelength differences are most heavily affected. To extinction correct the emission lines, we create maps of the observed Balmer ratio, $(\mathrm{H} \alpha / \mathrm{H} \beta)_{\text {obs }}$. We solve for $E(B-V)$ by using the following relation:

$$
E(B-V)=\log \left(\frac{(\mathrm{H} \alpha / \mathrm{H} \beta)_{\mathrm{obs}}}{(\mathrm{H} \alpha / \mathrm{H} \beta)_{\mathrm{int}}}\right) / 0.4[k(\mathrm{H} \beta)-\mathrm{k}(\mathrm{H} \alpha)]
$$

where $(\mathrm{H} \alpha / \mathrm{H} \beta)_{\text {int }}$ is the intrinsic ratio of 2.86 (Osterbrock 1989) assuming case B recombination. We use the Cardelli, Clayton \& Mathis (1989) extinction curve and assume a typical $R(V)$ value of 3.1 to determine $k$ values for $\mathrm{H} \alpha$ and $\mathrm{H} \beta$. We then use the calculated $E(B-V)$ to determine $\mathrm{A}(\lambda)$ at our emission line wavelengths to de-redden the emission line fluxes.

\subsection{Aliasing caused by differential atmospheric refraction}

As described in Green et al. (2017), differential atmospheric refraction (DAR) can combine with limited spatial sampling, as done in the SAMI survey to create aliasing effects on the spectra. The aliasing is caused by the atmosphere and is made worse by the way the SAMI instrument performs its drizzling to fill in gaps between fibres. While the overall DAR shift is accounted for, a combination of the seeing and sampling in the SAMI survey has meant that the 
DAR has introduced aliasing into the spectra on scales comparable to the point spread function (PSF). This aliasing is most noticeable when taking the ratio of two widely separated wavelength emission lines. With an oversampled PSF, we expect variations between neighbouring spaxels to be normally distributed. However, with aliasing, we find excess noise in flux ratios with wide wavelength separations. To correct for what is in effect a variation of the PSF with wavelength, when examining the Balmer decrement, Medling et al. (2018) smoothed the line ratio map by using a $5 \times 5$ spaxel Gaussian kernel with a full width at half-maximum of 1.6 spaxels ( 0.8 arcsec). This smoothing brings the noise down to levels we would expect with an oversampled PSF. We apply the same method not only to our Balmer decrement, but also to our metallicity and ionization parameter diagnostics $\left(\mathrm{R}_{23}, \mathrm{~N} 2 \mathrm{O} 2\right.$, and $\left.\mathrm{O} 32\right)$, as these all have a significant wavelength gap between emission lines.

\section{3 $\mathbf{R}_{23}$ diagnostic}

One of the most popular and well-calibrated strong emission line metallicity diagnostics is ([O $\left.\sim_{\mathrm{II}}\right] \lambda 3726, \lambda 3729+$ [O $\left.\left.\sim_{\mathrm{III}}\right] \lambda 4959, \lambda 5007\right) / \mathrm{H} \beta$, also known as $\mathrm{R}_{23}$, first introduced by Pagel et al. (1979). This diagnostic measures the sum of the two dominant ionization states of oxygen in HII regions, which captures the majority of the element. However, this diagnostic is sensitive to temperature and ionization, which has resulted in many $\mathrm{R}_{23}$ calibrations, each leading to different metallicity estimates (Pagel et al. 1979; Pagel, Edmunds \& Smith 1980; Edmunds \& Pagel 1984; McCall, Rybski \& Shields 1985; Dopita \& Evans 1986; TorresPeimbert, Peimbert \& Fierro 1989; McGaugh 1991; Zaritsky et al. 1994; Pilyugin 2000; Charlot \& Longhetti 2001; Kewley \& Dopita 2002; Kobulnicky \& Kewley 2004). For a comprehensive review and analysis of various metallicity calibrations, see Kewley \& Ellison (2008). Furthermore, due to this sensitivity to temperature, the $R_{23}$ diagnostic can be degenerate with both a high- and low-metallicity solution. Some $\mathrm{R}_{23}$ metallicity diagnostics take the ionization parameter into account (McGaugh 1991; Kewley \& Dopita 2002; Kobulnicky \& Kewley 2004). However, the determination of the ionization parameter is similarly difficult because many ionization parameter diagnostics have a significant dependence on metallicity. By using an iterative method described in KD02, we are able to constrain metallicity and ionization parameter simultaneously (see Section 3.5).

\subsection{2 diagnostic}

One way of measuring the ionization parameter is to measure the relative flux of emission lines from high- and low-ionization states of the same element. To determine the ionization parameter, we use the $\left[\mathrm{O}_{\text {III }}\right] \lambda 5007 /\left[\mathrm{O}_{\text {II }}\right] \lambda 3726, \lambda 3729$ (O32) diagnostic. KD02 and KK04 both presented theoretical calibrations for ionization parameter using the $\mathrm{O} 32$ diagnostic. However, the $\mathrm{O} 32$ diagnostic has a strong dependence on metallicity. Unlike the $\mathrm{R}_{23}$ diagnostic, the O32 diagnostic is unambiguous in the sense that it is not double valued except at high metallicities $\left(Z>2 Z_{\odot}\right)$. At lower metallicities, the polynomial fits to the theoretical relationship between ionization parameter and the $[\mathrm{O} \mathrm{III}] /\left[\mathrm{O}_{\mathrm{II}}\right]$ line ratio monotonically increase across the valid ionization parameter range.

\subsection{Iteration}

We determine the metallicity and ionization parameter simultaneously through an iterative process. We first use an initial metallic- ity estimate to constrain the $\mathrm{R}_{23}$ diagnostic to the upper or lower metallicity branch. The $[\mathrm{N} \mathrm{II]}] \lambda 6583 /[\mathrm{O}$ II] $] \lambda 3726, \lambda 3729$ (N2O2) diagnostic has very little dependence on ionization parameter (but is strongly affected by attenuation), and we use this diagnostic ratio for our initial metallicity estimate. For spaxels with $\mathrm{N} 2 \mathrm{O} 2<-1.2$, we place the spaxel on the lower $\mathrm{R}_{23}$ branch and assume a metallicity of $12+\log (\mathrm{O} / \mathrm{H})=8.2$ as the starting iteration point. For $\mathrm{N} 2 \mathrm{O} 2>-1.2$, we use the upper $\mathrm{R}_{23}$ branch and assume a metallicity of $12+\log (\mathrm{O} / \mathrm{H})=8.7$. Once an initial metallicity estimate has been determined, we use this value in the first estimate of the ionization parameter using the $\mathrm{O} 32$ diagnostic. This first ionization parameter estimate is then used to improve our metallicity estimate through the $\mathrm{R}_{23}$ diagnostic. We continue iterating between the $\mathrm{R}_{23}$ and $\mathrm{O} 32$ diagnostics until the metallicity and ionization parameter converge. We consider the metallicity and ionization parameter converged if the difference between iterations in the metallicity estimate is less than $0.1 \mathrm{dex}$ and the ionization parameter estimate is within $0.01 \mathrm{dex}$. We require this tolerance to be achieved for all spaxels used during analysis. The rate at which the metallicity and ionization parameter converge is usually proportional to the $\mathrm{S} / \mathrm{N}$ ratio. Spaxels with a $\mathrm{S} / \mathrm{N}>5$ in the used emission lines generally converge in $\sim 3$ iterations, while lower $\mathrm{S} / \mathrm{N}$ spaxels sometimes require $20+$ iterations. We impose an upper limit of 20 iterations to remove any non-converging spaxels from the maps. Spaxels that have not converged are discarded from the metallicity and ionization parameter maps.

\subsection{Error propagation}

The iterative method used to calculate the metallicity and ionization parameter makes it difficult to analytically propagate the error. To propagate line flux errors produced by LZIFU through to the metallicity and ionization parameter, we simulate 1000 maps for all emission lines used in the calculation. The maps are created such that the fluxes are Gaussian distributed with the LZIFU standard deviation for that emission line. Using the simulated line maps, metallicity and ionization parameter maps are created using the iterative process described in Section 3.5. The non-linearity of the resulting metallicity and ionization parameter diagnostics means that the metallicity and ionization parameter distributions are not necessarily Gaussian. To represent the spread of metallicity and ionization parameter, we determine the distance from the best-fitting value to the 16th and 84 th percentiles and calculate the average. This provides us with a measure of the uncertainty of the metallicity and ionization parameter maps, which are then propagated to the gradient errors.

\section{METALLICITY DISTRIBUTION}

We calculate metallicity and ionization parameter maps with their corresponding errors for our sample of 25 SAMI galaxies. In Fig. 2, we show two examples of the metallicity maps using different metallicity diagnostics accompanied by their error maps. In addition to the metallicity maps, we also show the Sloan Digital Sky Survey (SDSS) 3 colour image ( rri) of the galaxy with the SAMI field of view and its effective radius. The metallicity maps for the other 23 galaxies are presented in the Appendix. The majority of galaxies in our sample have metallicities in the range $8.5<12+\log (\mathrm{O} / \mathrm{H})<9.3$ in the radial ranges probed using the KK04 metallicity diagnostics. The mass-metallicity relation presented in Kewley \& Ellison (2008) shows that the nuclear metallicities for SDSS galaxies range between $8.7<12+\log (\mathrm{O} / \mathrm{H})<9.05$ for a mass range between $9.0<$ 


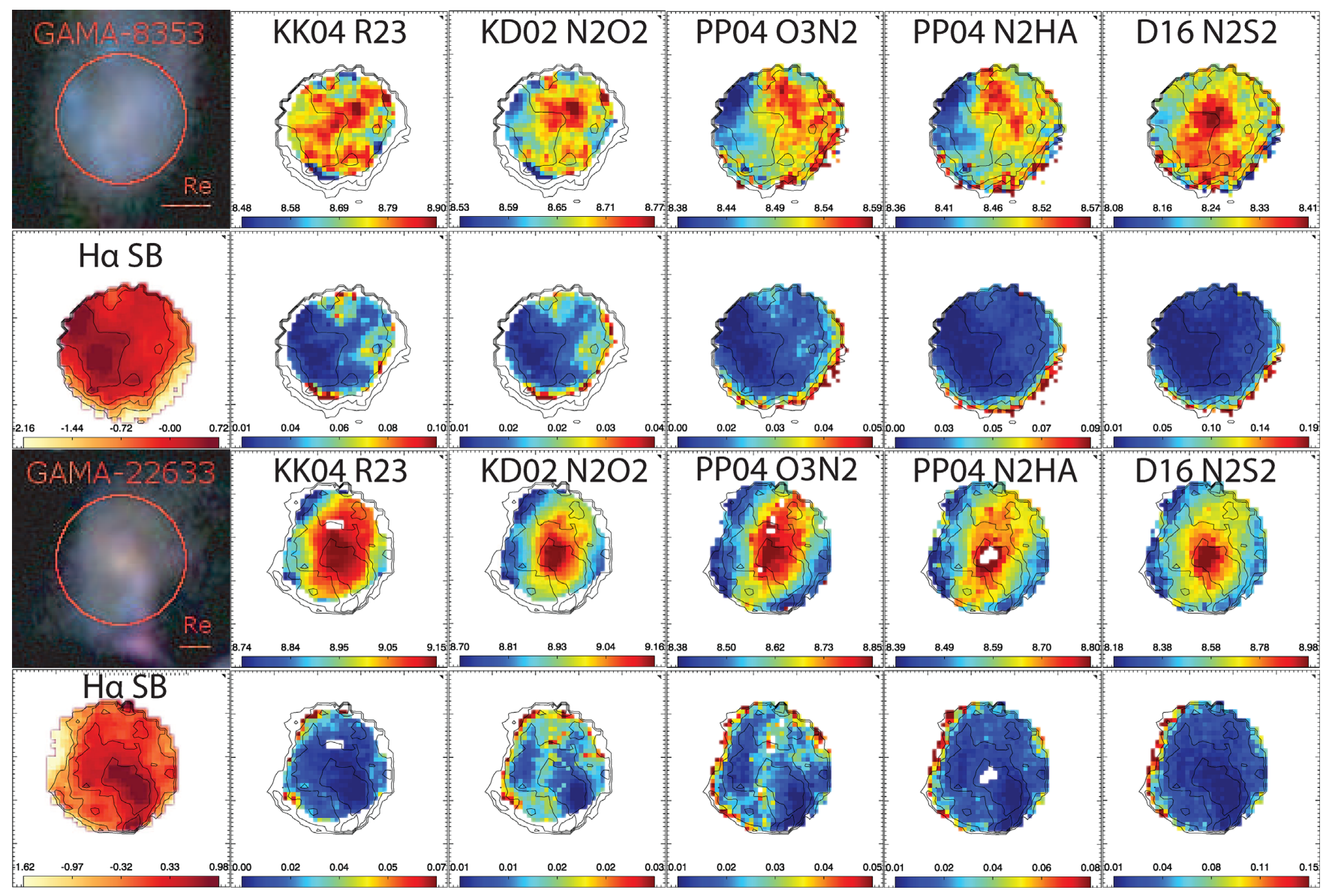

Figure 2. Each galaxy is presented as a $2 \times \$ 6$ grid. The top row of each grid shows the various metallicity maps with their corresponding error maps beneath them. Note that scale bars have been varied between different maps and galaxies in order to provide the best metallicity resolution possible. Column 1: SDSS composite image obtained from DR10. The red circles represent the 14.7 arcsec aperture of the SAMI hexabundle, and the scale bar shows the effective radius of the galaxy obtained from GAMA $R$ band. Below this, we show the $\mathrm{H} \alpha$ emission line map. We choose the $\mathrm{H} \alpha$ emission line map because we believe it provides the best representation of the galaxy structure and morphology. We overplot the H $\alpha$ contours onto each metallicity map to provide a point of reference when comparing metallicity diagnostics. Column 2: KK04 metallicity determined from the $\mathrm{R}_{23}$ line ratio. Column 3: KD02 metallicity determined from the N2O2 line ratio. Column 4: PP04 metallicity determined from the O3N2 line ratio. Column 5: PP04 metallicity determined from the N2HA line ratio. Column 6: D16 metallicity determined from the N2S2 line ratio. All metallicity maps are measured in units of $12+\log (\mathrm{O} / \mathrm{H})$.

$\log M_{*} / \mathrm{M}_{\odot}<10.5$. This is consistent with the metallicities within our sample for the same mass range, given that the SDSS fibre samples $\sim 20$ per cent of the galaxies' $B$-band light (Kewley, Jansen \& Geller 2005). The gas-phase metallicity increases over time. For the inside-out model of galaxy formation, we expect isolated galaxies to have strong negative metallicity gradients (Pagel \& Edmunds 1981; Edmunds \& Pagel 1984; Vilchez et al. 1988; Vila-Costas \& Edmunds 1992; Zaritsky et al. 1994). However, in interacting galaxies, the turbulent gas caused by the tidal forces stretches and flattens this metallicity gradient (Torrey et al. 2012). We find that in our sample, the majority of galaxies possess strong metallicity gradients (18/25), as expected for relatively isolated and undisturbed galaxies. We show these normalized metallicity gradients in Fig. 3 and provide a table of each linear fit in Table 2. For several galaxies, we also find a strong positive correlation between metallicity and SFR surface density, as shown in Fig. 4. This is consistent with several recent studies using SAMI data have shown SFR surface density gradients exist in the SAMI sample (Schaefer et al. 2017; Medling et al. 2018). Sánchez-Menguiano et al. (2016b) and Ho et al. (2017) showed that significant azimuthal variations exist in the metallicity distribution of NGC 6754 and
NGC 1365, respectively. However, we split each galaxy into quadrants and find little evidence of significant changes in the metallicity gradient, suggesting that at the spatial resolution of SAMI, spatial smoothing is sufficient to remove any traces of azimuthal variations, leaving only the radial gradients we observe. A resolution of at least $200 \mathrm{pc} / \mathrm{PSF}$ is needed to observe these azimuthal variations (Sánchez-Menguiano et al. 2016b). With a median redshift of $z=0.028$, an average seeing of 2.16 arcsec combined with the 0.8 arcsec smoothing to remove DAR, our galaxy sample has median resolution elements of $1.3 \mathrm{kpc} / \mathrm{PSF}$, much coarser than the minimum requirement found in Sánchez-Menguiano et al. (2016b). While statistically significant azimuthal variations are absent, there is evidence of a clumpy substructure in several metallicity maps (e.g. GAMA-8353 and GAMA-106717).

\subsection{Metallicity gradients}

While there is little azimuthal variation in the sample, there are clear radial gradients across our sample. The smoothness of these metallicity maps means that we are able to use a simple linear fit to the metallicity $(12+\log (\mathrm{O} / \mathrm{H}))$ as a function of radius. Sánchez- 


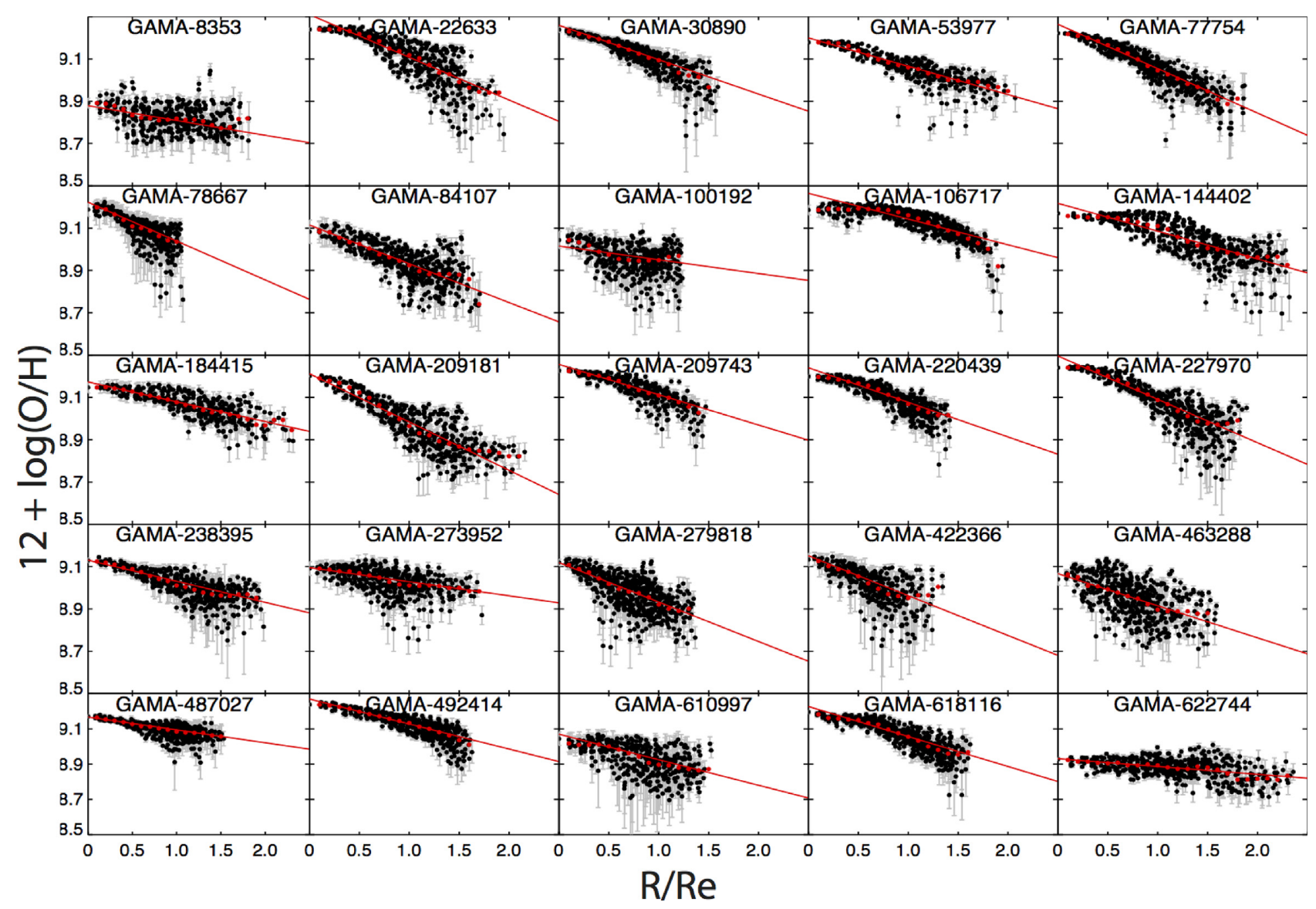

Figure 3. KK04 $\mathrm{R}_{23}$ metallicity gradients used in our analysis. We show $1 \sigma$ error bars for each spaxel, determined from the method described in Section 3.6. The best linear fit to the metallicity gradient is given as a red line. The median metallicity in bins of $0.1 R / R_{\mathrm{e}}$ are filled red circles. The results are summarized in Table 2.

Menguiano et al. (2018) showed that broken linear fits can also be used to describe the metallicity gradients of MUSE galaxies. Broken linear fits allow for the fitting of steepening or flattening metallicity gradients, resulting in a more robust fit for metallicity gradients that vary with radii. For this study, we use single linear fits to the galaxy metallicity gradients. We determine the radial distance of each pixel from the centre, taking into account the ellipticity and position angle of the object. We also normalize the radius by the size of its disc using its effective radius $\left(R_{\mathrm{e}}\right)$ in the $R$ band measured using GALFIT (Peng et al. 2002) by the GAMA survey (Kelvin et al. 2012). This removes the size dependence that the metallicity gradient has when measured on a physical scale (Sánchez et al. 2014; Ho et al. 2015). We use the robust line fitting routine LTS_LINEFIT (Cappellari et al. 2013) to fit a linear trend to the data. We choose LTS_LINEFIT for its ability to identify and separate outliers from the input data as well as provide standard errors to the output fit parameters. To calculate the standard errors on the output fit parameters, we provide LTS_LINEFIT with the metallicity errors calculated from the method described in Section 3.6. We show the radial metallicity gradients of our sample in Fig. 3 along with the best linear fit and radially binned median points. The Pearson correlation coefficient (PCC) is a measure of the presence of a linear trend. A magnitude of greater than 0.6 is usually accepted as a strong indication of a linear trend. The majority of radial metallicity gradients determined by LTS_LINEFIT show a strong trend (PCC magnitude $>0.6$ ), with four galaxies presenting with very strong PCC (magnitude $>0.8$ ). Fig. 5 shows the normalized metallicity gradients of galaxies against their stellar masses. Within our mass range of $9.0<\log \left(M_{*} / \mathrm{M}_{\odot}\right)<10.5$, the normalized metallicity gradients range from -0.20 to $-0.03 \mathrm{dex} / R_{\mathrm{e}}$. There appears to be a slight correlation with steeper metallicity gradients occurring at higher masses. We fit the relationship with a linear trend and find a slope of $-0.065 \pm 0.021 \mathrm{dex} / R_{\mathrm{e}} / \log \left(M_{*} / \mathrm{M}_{\odot}\right)$ with a PCC of -0.54 . Belfiore et al. (2017) find a similar trend with steeper metallicity gradients occurring in more-massive galaxies in the mass range $9.0<\log \left(M_{*} / \mathrm{M}_{\odot}\right)<10.5$. Estimating the error on the PCC through bootstrapping analysis, we find PCC = $-0.54 \pm 0.06$ for the relationship between stellar mass and metallicity gradients. This indicates that there exists a weak negative linear trend between stellar mass and metallicity gradients for galaxies in the mass range $9.0<\log \left(M_{*} / \mathrm{M}_{\odot}\right)<10.5$. This disagrees with previous studies by Sánchez et al. (2012, 2014), Ho et al. (2015), and Sánchez-Menguiano et al. (2016a), who found no variation in radial metallicity gradients in their sample when normalized with either $\mathrm{R}_{25}$ or $R_{\mathrm{e}}$.

\subsection{Mass-metallicity relation}

While the radial metallicity gradients appear to be weakly dependent on galaxy masses, across $9.0<\log \left(M_{*} / \mathrm{M}_{\odot}\right)<10.5$ there still exists the global mass-metallicity relation. Fig. 6 shows the correlation of the metallicity intercept with stellar mass for multiple metallicity 
Table 2. List of metallicity gradients and intercepts with their $1 \sigma$ uncertainties, root mean square (rms) scatter, and PCC values.

\begin{tabular}{lcccc}
\hline GAMA ID & $\begin{array}{c}\text { Central metallicity } \\
(12+\log (\mathrm{O} / \mathrm{H}))\end{array}$ & $\begin{array}{c}\text { Gradient } \\
\left(\mathrm{dex} / R_{\mathrm{e}}\right)\end{array}$ & $\mathrm{rms}$ & $\mathrm{PCC}$ \\
\hline 008353 & $8.831 \pm \$ 0.007$ & $-0.061 \pm \$ 0.007$ & 0.081 & -0.33 \\
022633 & $9.209 \pm \$ 0.006$ & $-0.177 \pm \$ 0.006$ & 0.056 & -0.71 \\
030890 & $9.164 \pm \$ 0.002$ & $-0.142 \pm \$ 0.003$ & 0.034 & -0.87 \\
053977 & $9.112 \pm \$ 0.003$ & $-0.117 \pm \$ 0.003$ & 0.027 & -0.69 \\
077754 & $9.169 \pm \$ 0.002$ & $-0.184 \pm \$ 0.003$ & 0.032 & -0.89 \\
078667 & $9.133 \pm \$ 0.004$ & $-0.161 \pm \$ 0.006$ & 0.037 & -0.70 \\
084107 & $9.038 \pm \$ 0.006$ & $-0.160 \pm \$ 0.006$ & 0.047 & -0.65 \\
100192 & $8.951 \pm \$ 0.007$ & $-0.057 \pm \$ 0.008$ & 0.061 & -0.33 \\
106717 & $9.169 \pm \$ 0.003$ & $-0.106 \pm \$ 0.003$ & 0.030 & -0.72 \\
144402 & $9.128 \pm \$ 0.006$ & $-0.115 \pm \$ 0.004$ & 0.049 & -0.68 \\
184415 & $9.089 \pm \$ 0.004$ & $-0.082 \pm \$ 0.004$ & 0.033 & -0.76 \\
209181 & $9.123 \pm \$ 0.007$ & $-0.200 \pm \$ 0.006$ & 0.073 & -0.77 \\
209743 & $9.162 \pm \$ 0.003$ & $-0.125 \pm \$ 0.004$ & 0.029 & -0.84 \\
220439 & $9.148 \pm \$ 0.003$ & $-0.143 \pm \$ 0.003$ & 0.027 & -0.79 \\
227970 & $9.196 \pm \$ 0.005$ & $-0.179 \pm \$ 0.004$ & 0.065 & -0.75 \\
238395 & $9.052 \pm \$ 0.003$ & $-0.087 \pm \$ 0.003$ & 0.038 & -0.72 \\
273952 & $9.020 \pm \$ 0.004$ & $-0.058 \pm \$ 0.005$ & 0.041 & -0.44 \\
279818 & $9.042 \pm \$ 0.005$ & $-0.163 \pm \$ 0.007$ & 0.073 & -0.30 \\
422366 & $9.070 \pm \$ 0.004$ & $-0.165 \pm \$ 0.007$ & 0.067 & -0.61 \\
463288 & $8.996 \pm \$ 0.008$ & $-0.133 \pm \$ 0.009$ & 0.080 & -0.48 \\
487027 & $9.084 \pm \$ 0.002$ & $-0.064 \pm \$ 0.002$ & 0.025 & -0.65 \\
492414 & $9.173 \pm \$ 0.003$ & $-0.124 \pm \$ 0.003$ & 0.032 & -0.84 \\
610997 & $8.999 \pm \$ 0.007$ & $-0.127 \pm \$ 0.008$ & 0.082 & -0.51 \\
618116 & $9.136 \pm \$ 0.003$ & $-0.149 \pm \$ 0.004$ & 0.036 & -0.78 \\
622744 & $8.877 \pm \$ 0.004$ & $-0.039 \pm \$ 0.003$ & 0.048 & -0.47 \\
\hline & & & &
\end{tabular}

Table 3. List of ionization parameter gradients and intercepts with their $1 \sigma$ uncertainties, rms scatter, and PCC values.

\begin{tabular}{lcrrr}
\hline GAMA ID & $\begin{array}{c}\text { Central ionization } \\
(\log (q))\end{array}$ & $\begin{array}{c}\text { Gradient } \\
\left(\mathrm{dex} / R_{\mathrm{e}}\right)\end{array}$ & $\mathrm{rms}$ & \multicolumn{1}{c}{ PCC } \\
\hline 008353 & $7.210 \pm \$ 0.011$ & $-0.017 \pm \$ 0.010$ & 0.086 & -0.08 \\
022633 & $7.392 \pm \$ 0.010$ & $-0.022 \pm \$ 0.009$ & 0.081 & -0.12 \\
030890 & $7.329 \pm \$ 0.006$ & $-0.039 \pm \$ 0.008$ & 0.059 & 0.00 \\
053977 & $7.252 \pm \$ 0.005$ & $-0.047 \pm \$ 0.005$ & 0.038 & -0.43 \\
077754 & $7.236 \pm \$ 0.005$ & $0.024 \pm \$ 0.006$ & 0.051 & 0.18 \\
078667 & $7.270 \pm \$ 0.009$ & $-0.010 \pm \$ 0.013$ & 0.060 & 0.08 \\
084107 & $7.410 \pm \$ 0.012$ & $-0.108 \pm \$ 0.011$ & 0.091 & -0.37 \\
100192 & $7.286 \pm \$ 0.012$ & $0.059 \pm \$ 0.015$ & 0.084 & 0.24 \\
106717 & $7.344 \pm \$ 0.006$ & $-0.010 \pm \$ 0.005$ & 0.048 & -0.16 \\
144402 & $7.312 \pm \$ 0.008$ & $-0.052 \pm \$ 0.005$ & 0.063 & -0.40 \\
184415 & $7.247 \pm \$ 0.008$ & $0.014 \pm \$ 0.007$ & 0.058 & 0.08 \\
209181 & $7.263 \pm \$ 0.009$ & $-0.018 \pm \$ 0.008$ & 0.072 & 0.03 \\
209743 & $7.284 \pm \$ 0.007$ & $-0.029 \pm \$ 0.009$ & 0.057 & -0.21 \\
220439 & $7.209 \pm \$ 0.006$ & $0.026 \pm \$ 0.007$ & 0.052 & 0.12 \\
227970 & $7.230 \pm \$ 0.012$ & $0.063 \pm \$ 0.011$ & 0.095 & 0.25 \\
238395 & $7.291 \pm \$ 0.007$ & $0.013 \pm \$ 0.006$ & 0.065 & 0.07 \\
273952 & $7.306 \pm \$ 0.010$ & $0.066 \pm \$ 0.011$ & 0.086 & 0.29 \\
279818 & $7.299 \pm \$ 0.008$ & $0.048 \pm \$ 0.010$ & 0.072 & 0.11 \\
422366 & $7.303 \pm \$ 0.014$ & $0.006 \pm \$ 0.020$ & 0.109 & 0.01 \\
463288 & $7.370 \pm \$ 0.015$ & $-0.006 \pm \$ 0.018$ & 0.135 & 0.03 \\
487027 & $7.205 \pm \$ 0.004$ & $-0.033 \pm \$ 0.006$ & 0.043 & -0.12 \\
492414 & $7.312 \pm \$ 0.007$ & $0.032 \pm \$ 0.007$ & 0.059 & 0.09 \\
610997 & $7.239 \pm \$ 0.011$ & $-0.025 \pm \$ 0.012$ & 0.087 & -0.03 \\
618116 & $7.229 \pm \$ 0.006$ & $0.010 \pm \$ 0.006$ & 0.055 & 0.09 \\
622744 & $7.581 \pm \$ 0.006$ & $-0.144 \pm \$ 0.005$ & 0.062 & -0.73 \\
\hline & & & & \\
\hline & & & & \\
& & & & \\
& & & & \\
& & &
\end{tabular}

diagnostics. Kewley \& Ellison (2008) provide fits to the massmetallicity relation for a range of different metallicity diagnostics. We plot the mass-metallicity fit for several metallicity diagnostics as the dotted red line in Fig. 6. There is a clear offset between the massmetallicity fit and the metallicity intercepts caused by using the central interpolated metallicities rather than aperture metallicities. We fit these offsets using MPFIT (Markwardt 2009) and show the best least-squares fit to the interpolated metallicities. Similar trends with the metallicity intercept were found in Sánchez et al. (2014), who also attributed it to the mass-metallicity relation.

\section{IONIZATION PARAMETER DISTRIBUTION}

\subsection{Ionization parameter gradients}

In contrast to the metallicity maps, the ionization parameter maps (Fig. 7) show no clear radial or azimuthal trends. Instead, we see a range of different distributions ranging from weak gradients, flat maps, and clumpy distributions. The majority of galaxies tend to have ionization parameters in the range $7.0<\log \left(q\left[\mathrm{~cm} \mathrm{~s}^{-1}\right]\right)<$ 7.8. We measure the radial ionization parameter gradients of the galaxies using robust line fits in the same way as the metallicity gradients. The ionization parameter radial gradients are presented in Fig. 8fig8.fig and compared to stellar mass in Fig. 9. We summarise the ionization parameter gradients in Table 3. All galaxies except three have a PCC magnitude of less than 0.4 , indicating very weak significance of these linear fits. GAMA-622744 appears to be the only galaxy with a significant ionization parameter gradient (PCC magnitude $=0.73$ ). Kaplan et al. (2016) found significant ionization parameter gradients in their sample of eight galaxies using VENGA data. The galaxies in their sample were chosen to have significant and highly resolved bulges. Kaplan et al. (2016) used the same $\mathrm{O} 32$ ionization parameter diagnostics from KK04 as we do but use one iteration rather than a convergence condition when calculating ionization parameter. Both methods provide them with similar results. The distribution of ionization parameter in their maps follows the distribution of SFR surface density in many of their galaxies and show strong radial gradients.

\subsection{Ionization parameter and galaxy properties}

While we see no significant radial or azimuthal trends in the ionization parameter for most of our sample, GAMA-8353 and GAMA-22633 show patterns in $q$ that are suggestive of the spiral arm features seen in the associated three-colour and $\mathrm{H} \alpha$ maps in each galaxy. Such an association could indicate that the ionization parameter is larger in areas of high star formation, a trend seen by Dopita et al. (2014) in a sample of luminous infrared galaxies above a threshold ionization parameter $\left(\log \left(q\left[\mathrm{~cm} \mathrm{~s}^{-1}\right]\right)\right.$ $>7.2$ - 7.4). Dopita et al. (2014) quantified this relation as $q\left[\mathrm{~cm} \mathrm{~s}^{-1}\right] \propto \mathrm{SFR}\left[\mathrm{M}_{\odot} \text { year }^{-1} \mathrm{kpc}^{-2}\right]^{0.34 \pm 0.08}$. Using SFR surface density maps created by Medling et al. (2018), we find that 71 per cent $(17 / 24)$ of galaxies present a slight positive correlation between SFR surface density and ionization parameter (Fig. 10). However, the strength of these gradients is weak with only GAMA622744 having a PCC magnitude of greater than 0.6 . We also investigate how the ionization parameter varies with metallicity (Fig. 11). We do this by plotting the KD02 metallicity determined from the $\mathrm{N} 2 \mathrm{O} 2$ diagnostic against the KK04 ionization parameter measurements. We use the KD02 N2O2 metallicity diagnostic instead of the KK04 R23 metallicity diagnostic to try and exclude any possible dependences between the two parameters caused by the iterative 


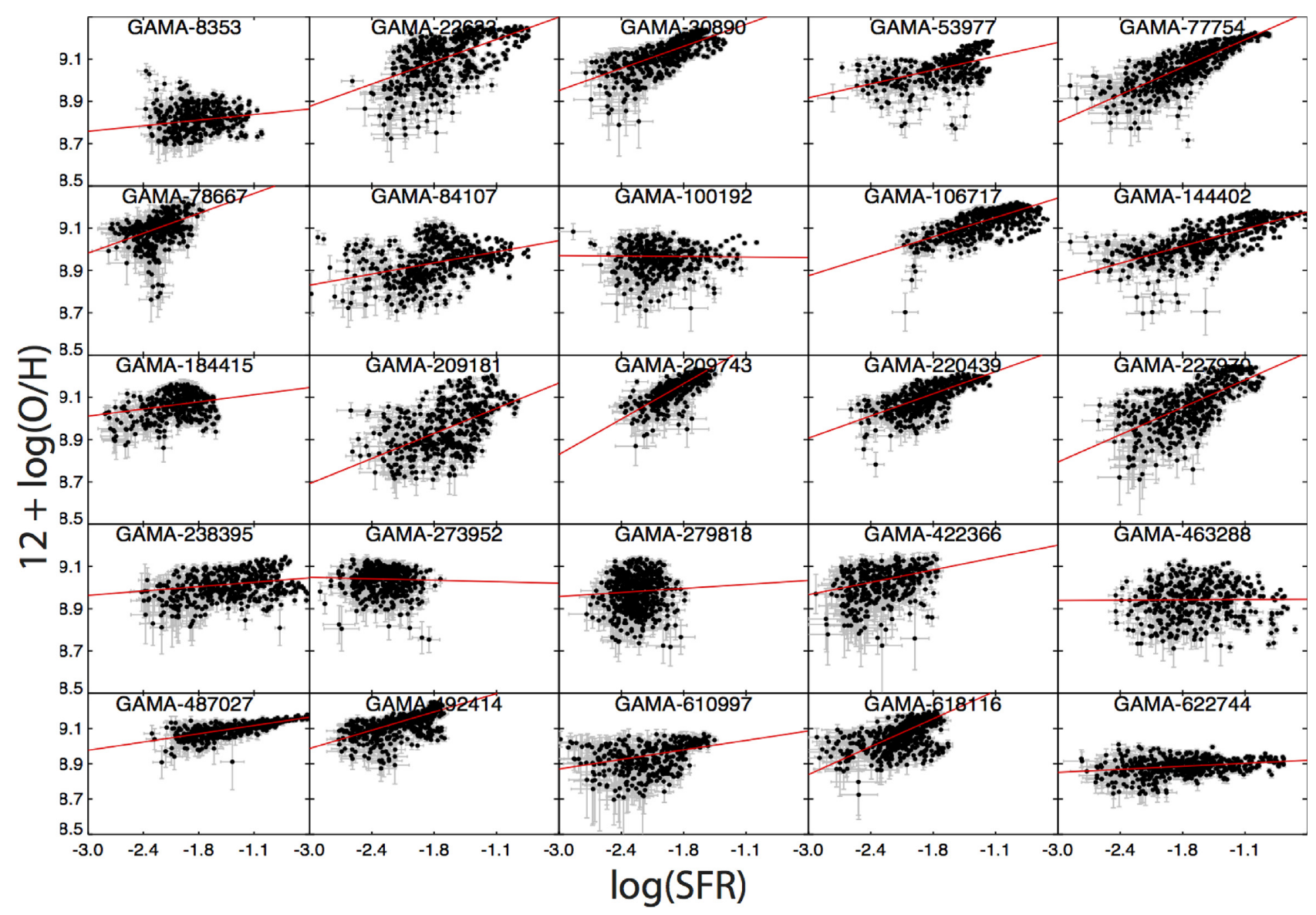

Figure 4. KK04 $\mathrm{R}_{23}$ metallicity as a function of SFR surface density. We obtain maps of SFR surface density in units of $\mathrm{M}_{\odot}$ year $^{-1} \mathrm{kpc}^{-2}$ from Medling et al. (2018). We show the best linear fit as a red line and summarize the results in Table C1.

method used to calculate the ionization parameter. Again, we find that only GAMA-622744 produces a significant PCC. The correlation between metallicity and ionization parameter for GAMA622744 is likely driven by the fact that it is the only galaxy in our sample with a significant negative ionization parameter gradient and not necessarily because of an intrinsic correlation between metallicity and ionization parameter. Dopita et al. (2014) found a strong positive trend between the metallicity and ionization parameter, which is not seen in either this work or Kaplan et al. (2016). Dopita et al. (2006) provide a theoretical relationship between gas-phase metallicity and ionization parameter, $q\left[\mathrm{~cm} \mathrm{~s}^{-1}\right] \propto \mathrm{Z}[\mathrm{O} / \mathrm{H}]^{-0.8}$.

\section{DISCUSSION}

\subsection{Metallicity gradients}

Using the KK04 $\mathrm{R}_{23}$ strong line emission diagnostic, we find a weak dependence in the slope of the normalized radial metallicity gradient with the stellar mass of the galaxy. This is inconsistent with the results found by several other recent studies on radial metallicity gradients in galaxies (Sánchez et al. 2012, 2014; Ho et al. 2015; Sánchez-Menguiano et al. 2016a). However, as demonstrated by Kewley \& Ellison (2008), the calculated metallicities are strongly dependent upon the calibration used. Based on this, the derived metallicity gradients may also depend upon the particular diagnostic used. Belfiore et al. (2017) calculated metallicity gradients for galaxies using a diagnostic derived from the $\mathrm{R}_{23}$ line ratio and also found a dependence on metallicity gradients with stellar mass. Although Belfiore et al. (2017) use the same $\mathrm{R}_{23}$ diagnostic, they use the Maiolino et al. (2008) calibration to determine metallicities, making a direct comparison between results difficult. We find a mean metallicity gradient value of $-0.12 \mathrm{dex} / R_{\mathrm{e}}$ with a standard deviation of 0.05 using the KK04 $\mathrm{R}_{23}$ metallicity diagnostic. Since there appears to be a dependence on metallicity gradients with stellar mass, sample selection plays an important role in the determination of mean metallicity gradients. Although Belfiore et al. (2017) use a different metallicity calibration to the $\mathrm{R}_{23}$ diagnostic, we note that they seem to find a shallower, although still consistent, mean metallicity gradient $(-0.08 \pm 0.12$ $\operatorname{dex} / R_{\mathrm{e}}$ ) than the ones determined here. The shallower mean metallicity gradient is caused by differences in sample selection. Belfiore et al. (2017) sample a wider stellar mass range, including relatively more low-mass galaxies. Since metallicity gradients have a stellar mass dependence, these lower mass galaxies have shallower metallicity gradients and hence decrease the mean metallicity gradient of the sample. This effect is also demonstrated by Belfiore et al. (2017) with a shallower volume-limited mean metallicity gradient, where low-mass galaxies are relatively heavier weighted. Sánchez et al. (2012, 2014), and Sánchez-Menguiano et al. (2016a) used the PP04 O3N2 diagnostic with their sample of CALIFA galaxies in order to analyse the metallicity gradients of galaxies, and Sánchez-Menguiano et al. (2016a) found a mean metallicity gradient of $-0.11 \pm 0.07 \mathrm{dex} / R_{\mathrm{e}}$. We recalculate our gradients using the PP04 O3N2 diagnostic and find a mean metallicity gradient of 


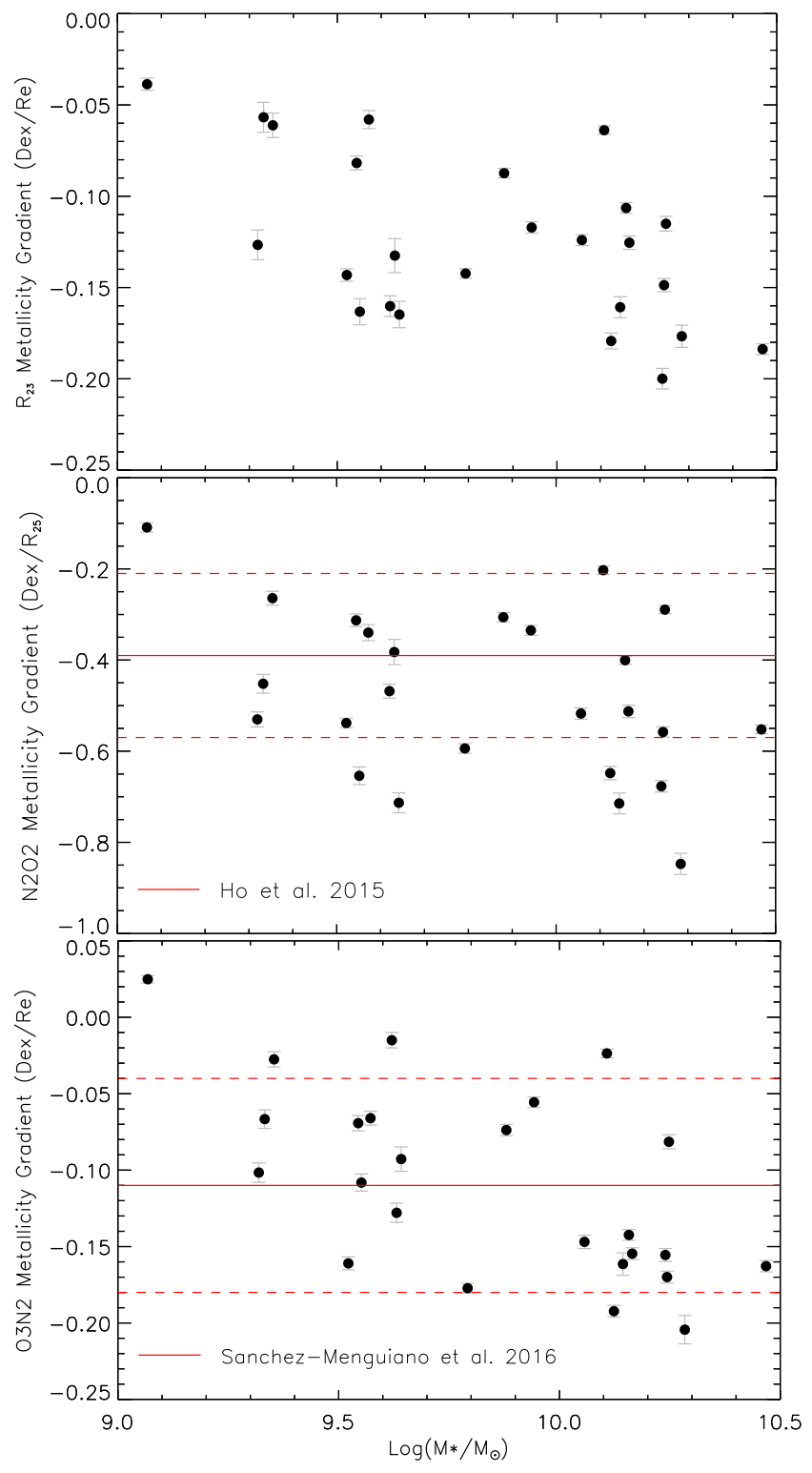

Figure 5. Normalized metallicity gradients as a function of mass using three common metallicity diagnostics. For the KD02 and PP04 metallicity diagnostics, we compare the results presented in Ho et al. (2015) and Sánchez-Menguiano et al. (2016a). The solid red line shows the mean metallicity gradient with $1 \sigma$ scatter shown as dotted red lines.

$-0.10 \pm 0.06 \mathrm{dex} / R_{\mathrm{e}}$ after excluding the inner $\left(R / R_{\mathrm{e}}<0.5\right)$ and outer sections $\left(R / R_{\mathrm{e}}>2.0\right)$ of the galaxies in the same way as Sánchez et al. (2012, 2014) and Sánchez-Menguiano et al. (2016a). Our metallicity gradients are consistent with those presented in all three studies. Our results are also consistent with the PP04 O3N2 metallicity gradients presented in Belfiore et al. (2017), which found a mean metallicity gradient of $-0.08 \pm 0.10 \mathrm{dex} / R_{\mathrm{e}}$. Belfiore et al. (2017) again present with slightly shallower but still consistent mean metallicity gradient. Belfiore et al. (2017) also find a mass dependence of the $\mathrm{O} 3 \mathrm{~N} 2$ metallicity gradients, meaning their wider stellar mass range may explain their slightly shallower mean metallicity gradient. For metallicity diagnostics that display mass-dependent metallicity gradients, sample selection appears to have a strong influence on the calculated mean metallicity gradient. Therefore, care
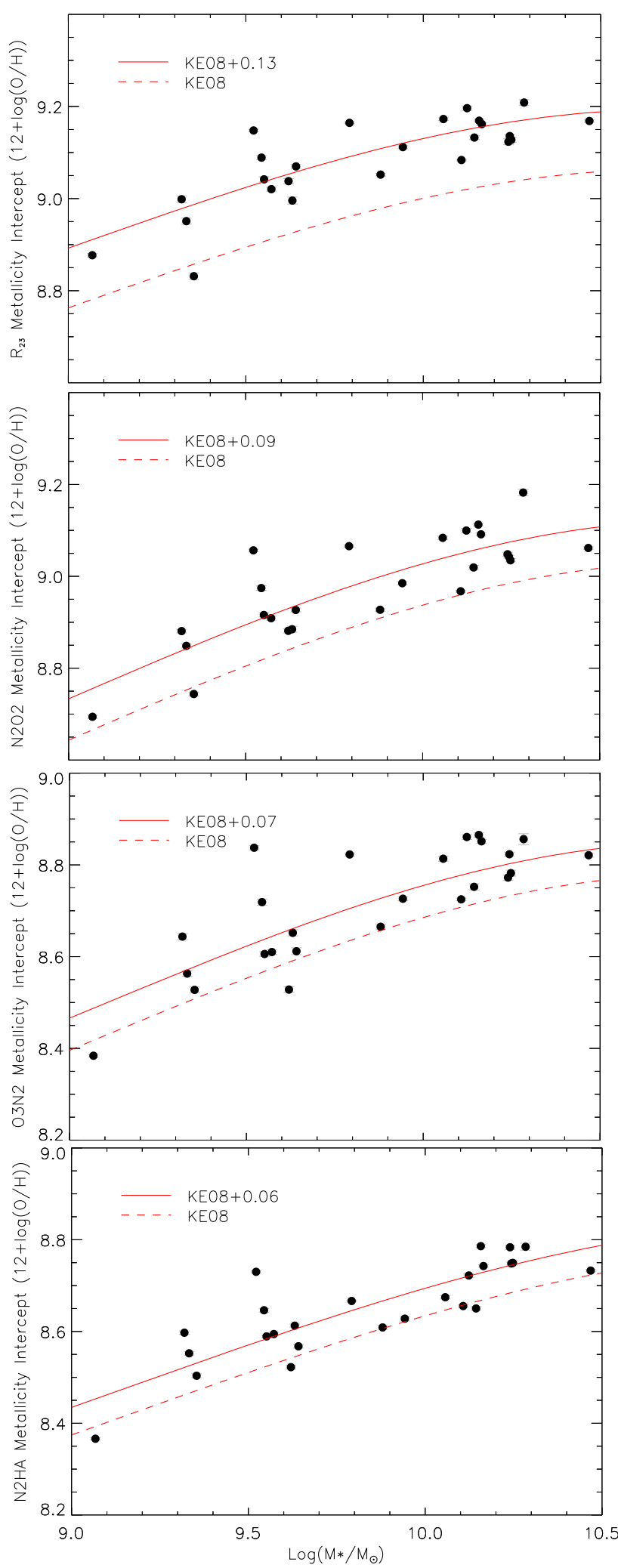

Figure 6. Metallicity intercepts as a function of mass for multiple metallicity diagnostics. We show the mass-metallicity relation for each diagnostic from Kewley \& Ellison (2008) as the dotted red line and fit an offset shown as the solid red line. 


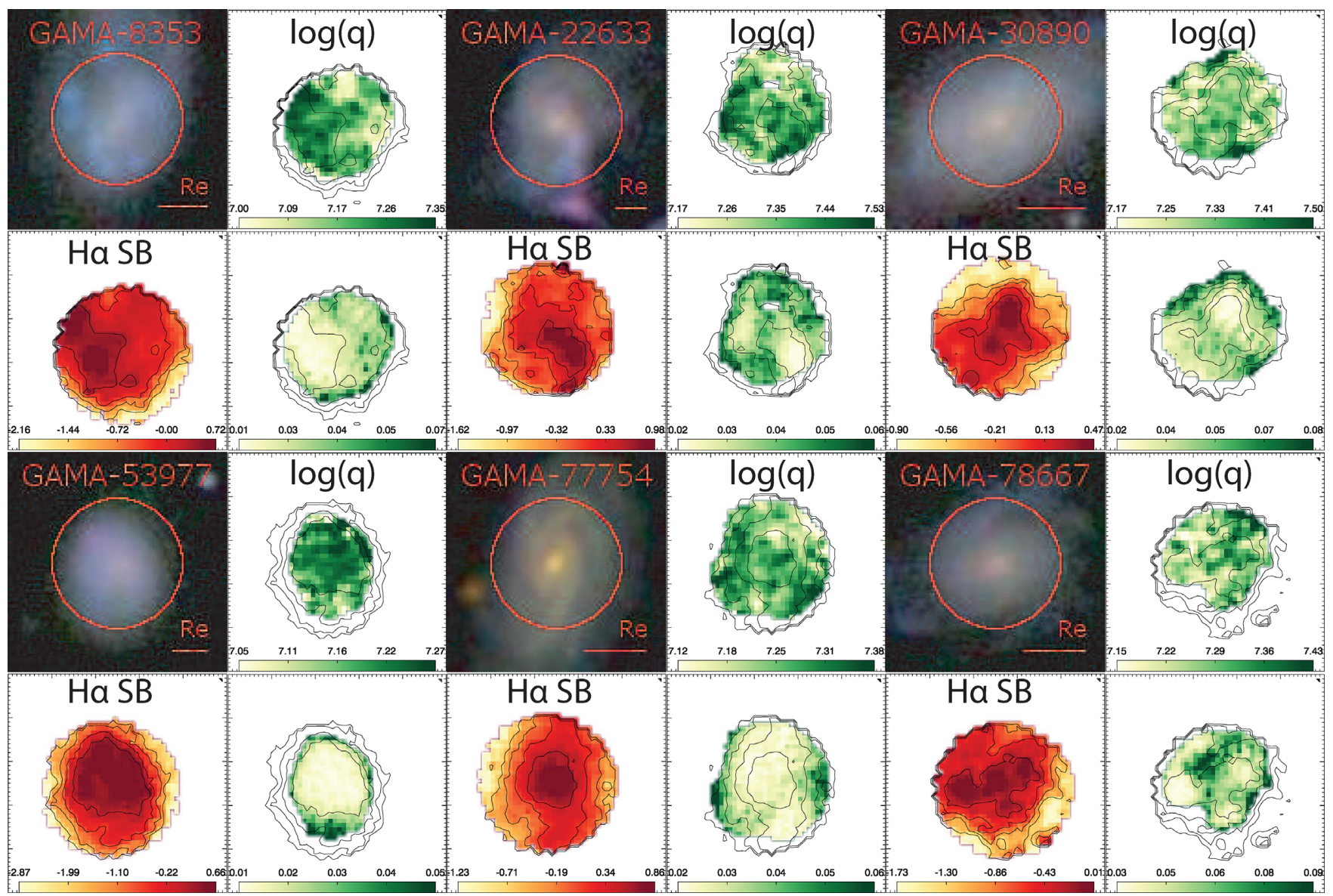

Figure 7. Each galaxy is presented with a $2 \times 2$ grid containing the results of our work. The top-left image of each grid contains the same SDSS image as Fig. 2. The H $\alpha$ emission line map with contours below the SDSS image is also identical to Fig. 2. To the right of each SDSS image is the ionization parameter map in units of $\log \left(\mathrm{cm} \mathrm{s}^{-1}\right)$ with overplotted $\mathrm{H} \alpha$ contours for comparison. Below each ionization parameter map is the associated error map, as described in Section 3.6. Note that scale bars have been varied between different maps and galaxies in order to provide the best resolution possible.

must be taken when comparing results between different studies as the stellar mass distribution of the sample may have a heavy impact on the results obtained. Sánchez et al. $(2012,2014)$ and SánchezMenguiano et al. (2016a) excluded the inner $\left(R / R_{\mathrm{e}}<0.5\right)$ and outer $\left(R / R_{\mathrm{e}}>2.0\right)$ galactic radii when measuring the metallicity gradients because of the observed flattening of the metallicity gradient that occurs at these radii (Bresolin et al. 2009; Rosales-Ortega et al. 2011; Bresolin, Kennicutt \& Ryan-Weber 2012; Marino et al. 2012; Sánchez et al. 2012, 2014; Sánchez-Menguiano et al. 2016a). We find metallicity gradient flattening occurring at $R / R_{\mathrm{e}}<0.5$ only for GAMA-106717 using either the PP04 O3N2 or KK04 diagnostic. Only two of our galaxies (GAMA-144402 and GAMA-622744) are observed beyond $2 R_{\mathrm{e}}$, and neither show any clear flattening of the metallicity gradient.

Ho et al. (2015) used the KD02 metallicity diagnostic to determine the metallicity gradients of a sample of CALIFA and WiFeS galaxies. Using the $\mathrm{R}_{25}$ scale length to normalize the metallicity gradients, Ho et al. (2015) found no significant dependence on stellar mass. Ho et al. (2015) found a mean metallicity gradient of $-0.39 \pm 0.18 \mathrm{dex} / \mathrm{R}_{25}$. We determine the metallicity gradients using the KD02 diagnostic, but the uncertainties in $\mathrm{R}_{25}$ for our sample were too large for a reliable comparison (based on values obtained from HyperLeda; Makarov et al. 2014). We instead assume a crude approximation of $\mathrm{R}_{25}=3.6 R_{\mathrm{e}}$ based on fits to $\mathrm{S} 0$ galaxies by
Williams, Bureau \& Cappellari (2009). Using this approximation, we obtain a mean metallicity gradient of $-0.48 \pm 0.18 \mathrm{dex} / \mathrm{R}_{25}$. Although the metallicity gradients agree within the errors, it is important to keep in mind that we have used only an approximation to $\mathrm{R}_{25}$ and have used the $R$-band scale length instead of the $B$-band, which was used in Ho et al. (2015).

\subsection{Scatter around metallicity gradients}

For the majority of metallicity gradients, the scatter increases noticeably at larger radii. Within $1 R_{\mathrm{e}}$, the standard deviation away from the metallicity gradient is approximately 0.04 dex and increases to 0.08 dex beyond $1 R_{\mathrm{e}}$. We find that this is driven mostly by the decrease in line flux, and hence $\mathrm{S} / \mathrm{N}$, at larger radii in the SAMI data. At an integrated $\mathrm{H} \alpha \mathrm{S} / \mathrm{N}<80$, the scatter is $0.07 \mathrm{dex}$, whereas at a $\mathrm{H} \alpha \mathrm{S} / \mathrm{N}>80$, the scatter decreases to about 0.03 dex. However, a decrease in $\mathrm{S} / \mathrm{N}$ does not account for all of the increase in scatter. In five of our galaxies, we notice that more than half of the spaxels within $1 R_{\mathrm{e}}$ have a $\mathrm{S} / \mathrm{N}<80$ and have significantly less scatter than those spaxels at radii larger than $1 R_{\mathrm{e}}$. We also notice a large bias of the scatter towards lower metallicities. We find that spaxels that deviate more than 0.1 dex from the metallicity gradient have an increased $R_{23}$ line ratio. All these spaxels also lie on the upper branch of the KK04 $\mathrm{R}_{23}$ metallicity diagnostic. The combina- 


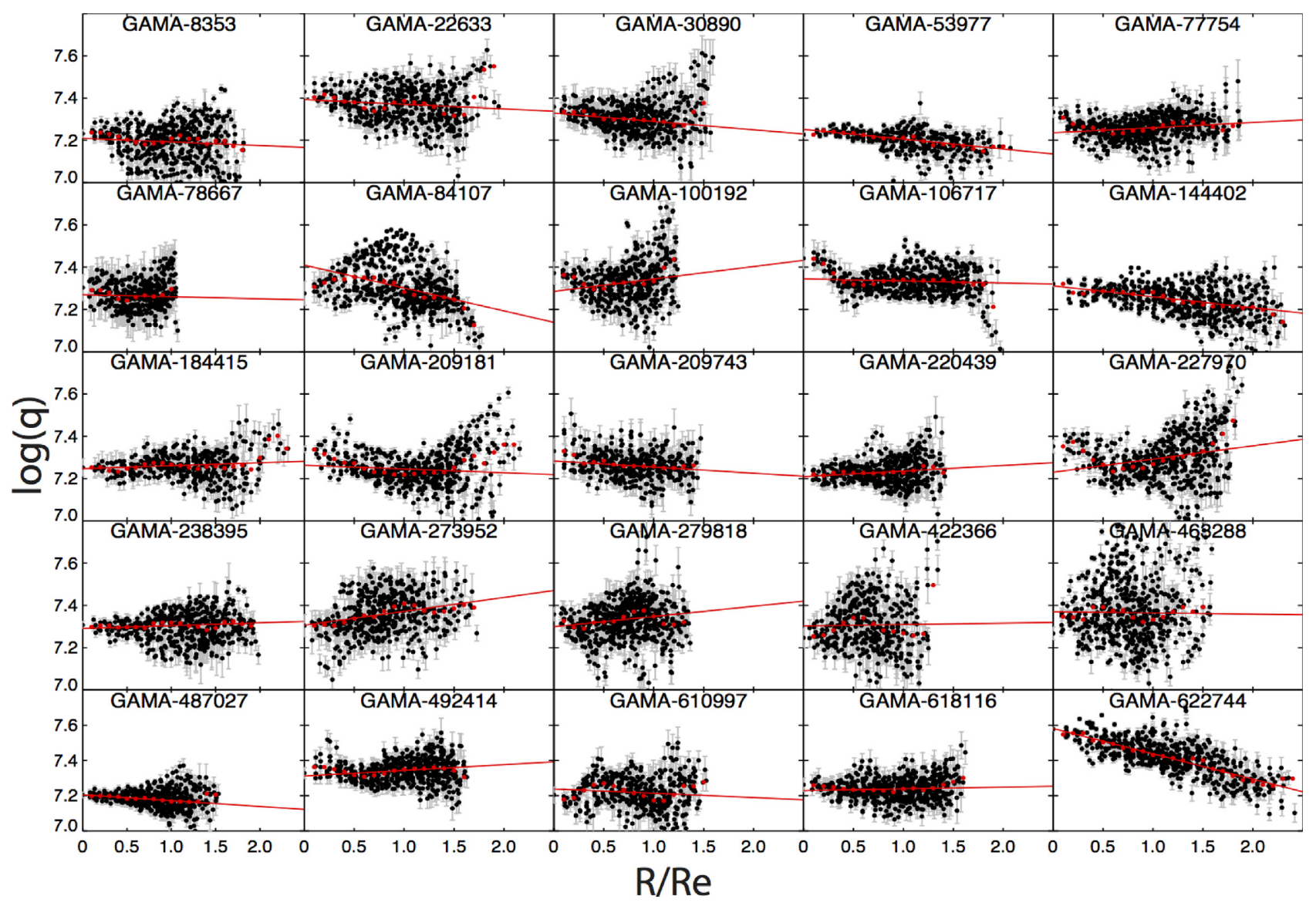

Figure 8. Same as Fig. 3 for ionization parameter. The results are summarized in Table 3 .

tion of these two effects leads to a lower metallicity measurement. In addition, the KK04 $\mathrm{R}_{23}$ metallicity diagnostic becomes less sensitive to metallicity at higher values of $R_{23}$, which only enhances this deviation. The larger $\mathrm{R}_{23}$ line ratio is caused by an increase in both the $\left[\mathrm{O}_{\mathrm{II}}\right] / \mathrm{H} \beta$ and $\left[\mathrm{O}_{\mathrm{III}}\right] / \mathrm{H} \beta$ line ratios. The $\left[\mathrm{O}_{\mathrm{II}}\right] / \mathrm{H} \beta$ line ratio has a larger percentage increase than the $[\mathrm{O} \mathrm{III}] / \mathrm{H} \beta$ line ratio. This leads to an overall decrease in the $\left[\mathrm{O}_{\mathrm{III}}\right] /\left[\mathrm{O}_{\mathrm{II}}\right]$ line ratio, causing lower ionization parameter measurements for a metallicity range of $7.6<12+\log (\mathrm{O} / \mathrm{H})<9.2$. One explanation for the enhanced line ratios at large radii is diffuse ionized gas (DIG) contamination. Using data from the MaNGA survey, Zhang et al. (2017) demonstrated the effects of DIG on emission line ratios and metallicity diagnostics. They found that the $[\mathrm{O} \mathrm{II}] / \mathrm{H} \beta$ line ratio is enhanced in DIG-dominated regions, while the DIG effects on the $[\mathrm{O}$ III] $] / \mathrm{H} \beta$ line ratio depend on the specific situation of the galaxy. In both cases, they also found a decrease in the $[\mathrm{O}$ III $] /[\mathrm{O}$ II $]$ line ratio.

\subsection{Mass-metallicity relation}

Fig. 6 shows the metallicity intercepts as a function of stellar mass with the mass-metallicity fit from Kewley \& Ellison (2008) shown as the dotted red line. A small positive offset of 0.13 was required to optimally fit the mass-metallicity relation to the intercept data; this is shown as the solid red line. This is to account for the fact that we are using the interpolated central metallicity, which simulates an infinitesimally small central aperture. The interpolated central metallicity would be systematically higher than the global metallicity or larger aperture metallicity measurements

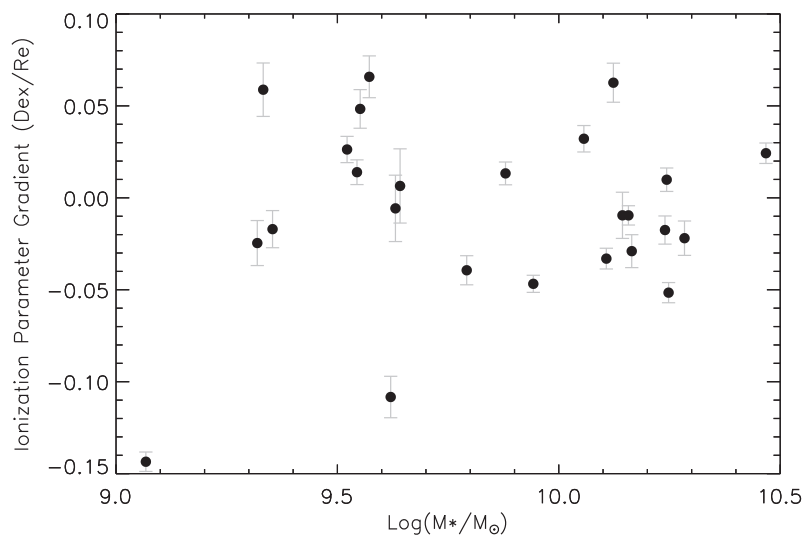

Figure 9. Normalized ionization parameter gradients using the KK04 O32 diagnostic as a function of stellar mass. We find no significant variation in the ionization parameter gradient as a function of galaxy mass.

because we are not averaging the regions of high and low metallicity. Tremonti et al. (2004) were able to simulate the effects of changing aperture metallicity measurements by showing that nearer galaxies had larger aperture metallicities than those further away of similar size. The nearer galaxies had a larger apparent size, meaning that they were restricted to sampling a smaller fraction of the galaxy. 


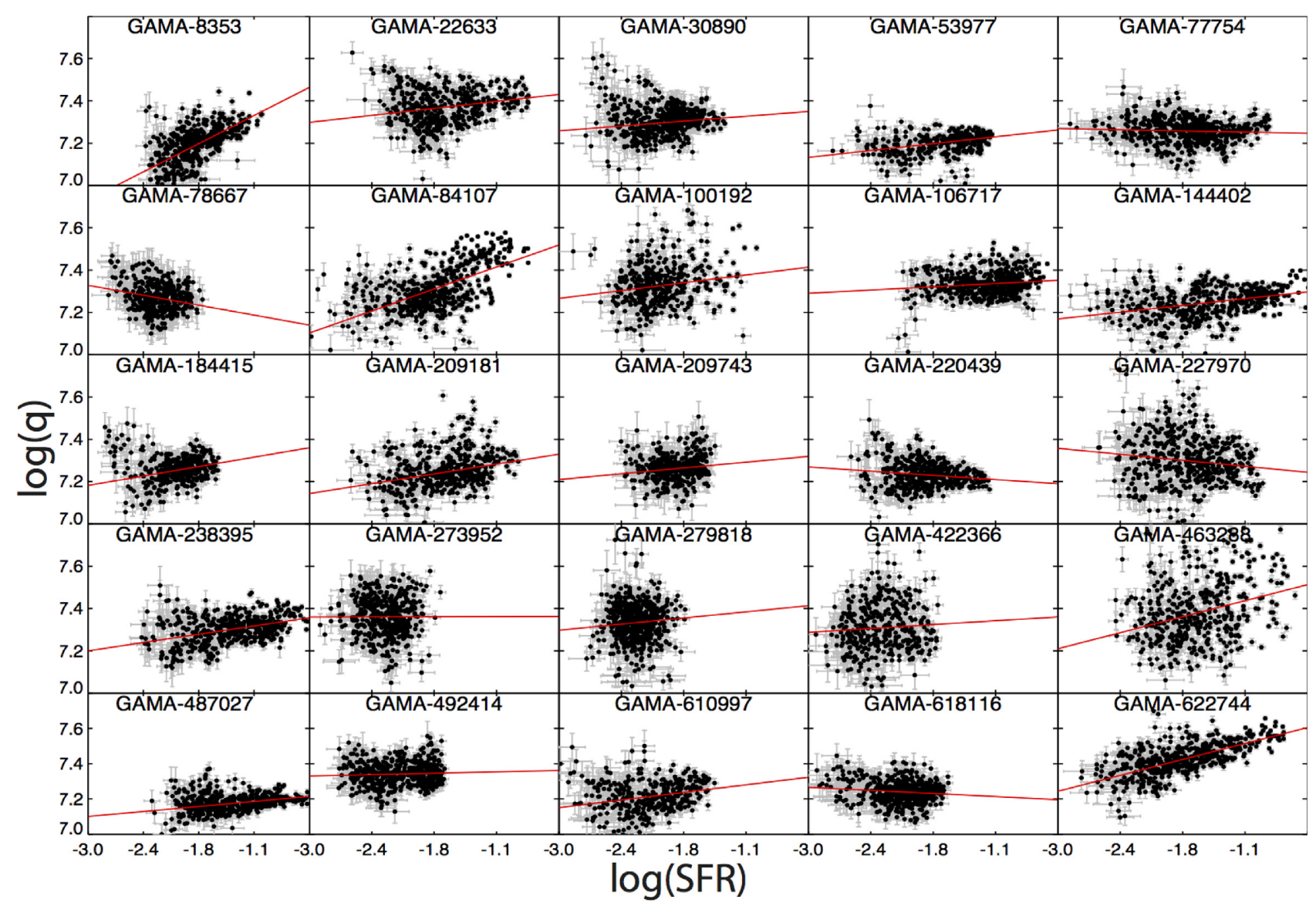

Figure 10. Same as Fig. 4 for ionization parameter. We summarize the results in Table C2.

\subsection{Ionization parameter gradients}

The ionization parameter maps produced by Kaplan et al. (2016) show significant radial ionization parameter gradients as well as a correlation with SFR. A correlation between the ionization parameter and SFR was also observed by Dopita et al. (2014) using a sample of WiFeS galaxies. Yuan, Kewley \& Rich (2013) and Mast et al. (2014) have shown that decreased spatial resolution leads to the flattening of observed metallicity gradients. Kaplan et al. (2016) has a median resolution of $387 \mathrm{pc}$, while the full SAMI survey has resolutions of the order of kpc. Our galaxy sample has a median spatial resolution of $1.3 \mathrm{kpc} / \mathrm{PSF}$ caused by the seeing limited observations with an average seeing of 2.16 arcsec and DAR smoothing of 0.8 arcsec. Our galaxies are significantly less massive and have a higher redshift, meaning that fine details are difficult to resolve compared to Kaplan et al. (2016). It is possible that the lack of ionization parameter gradients is due to the spatial smoothing caused by our inability to resolve the finer details due to limitations in seeing. More work using higher resolution data is needed in order to confirm if ionization parameter gradients are affected in the same way as metallicity gradients. The SAMI spectrograph does not have the spatial resolution required to resolve $\mathrm{H}_{\text {II }}$ regions at the redshift of the main galaxy survey. To obtain higher spatial resolution spectra of $\mathrm{H}$ II regions, a sister survey of nearby $\mathrm{H}$ II regions is being conducted in order to recalibrate the strong line emission diagnostics (SAMI Zoom, Sweet et al. in preparation). The galaxies used in Kaplan et al. (2016) are also more massive $\left(10.2<\log \left(M_{*} / \mathrm{M}_{\odot}\right)\right.$ $<11.6)$ than the mass range of the galaxies $\left(9.0<\log \left(M_{*} / \mathrm{M}_{\odot}\right)<\right.$ $10.5)$ used in this study. Although we find no variation in ionization parameter gradient or intercept with mass, the difference in galaxy masses could be a factor in the absence of ionization parameter gradients.

\subsection{Ionization parameter and galaxy properties}

Dopita et al. (2014) quantified the relationship between ionization parameter and $\mathrm{SFR}\left[\mathrm{M} \odot \mathrm{year}^{-1} \mathrm{kpc}^{-2}\right]$ as $q\left[\mathrm{~cm} \mathrm{~s}^{-1}\right] \propto \mathrm{SFR}\left[\mathrm{M}_{\odot} \text { year }^{-1} \mathrm{kpc}^{-2}\right]^{0.34} \pm 0.08$ when $\log \left(q\left[\mathrm{~cm} \mathrm{~s}^{-1}\right]\right)$ $\gtrsim 7.2-7.4$. From figure 13 of Dopita et al. (2014), we observe that below $\log \left(\operatorname{SFR}\left[\mathrm{M}_{\odot} \mathrm{year}^{-1} \mathrm{kpc}^{-2}\right]\right)<-0.5$, the correlation disappears and no trends are observed. Fig. 10 shows that all of our spaxels lie below $\log \left(\mathrm{SFR}\left[\mathrm{M}_{\odot}\right.\right.$ year $\left.\left.^{-1} \mathrm{kpc}^{-2}\right]\right)<-0.5$ with the large majority below $\log \left(\operatorname{SFR}\left[\mathrm{M}_{\odot}\right.\right.$ year $\left.\left.^{-1} \mathrm{kpc}^{-2}\right]\right)<-1.0$. We believe that this is the main reason that we do not observe the same trends as Dopita et al. (2014). The sSFR is even less correlated with ionization parameter, with PCC values consistently lower than those of SFR. GAMA-622744 is the only galaxy that displays a significant correlation between metallicity and ionization parameter. However, we believe this is not necessarily caused by an intrinsic relationship between metallicity and ionization parameter, but rather because GAMA-622744 is the only galaxy that possess a significant ionization parameter gradient. The positive correlation contradicts the theoretical relation presented in Dopita et al. (2006) $\left(q\left[\mathrm{~cm} \mathrm{~s}^{-1}\right] \propto Z[\mathrm{O} / \mathrm{H}]^{-0.8}\right)$. Many of the galaxies in Dopita et al. (2014) show a positive correlation between ionization parameter and metallicity, while our work lacks any significant trends. 


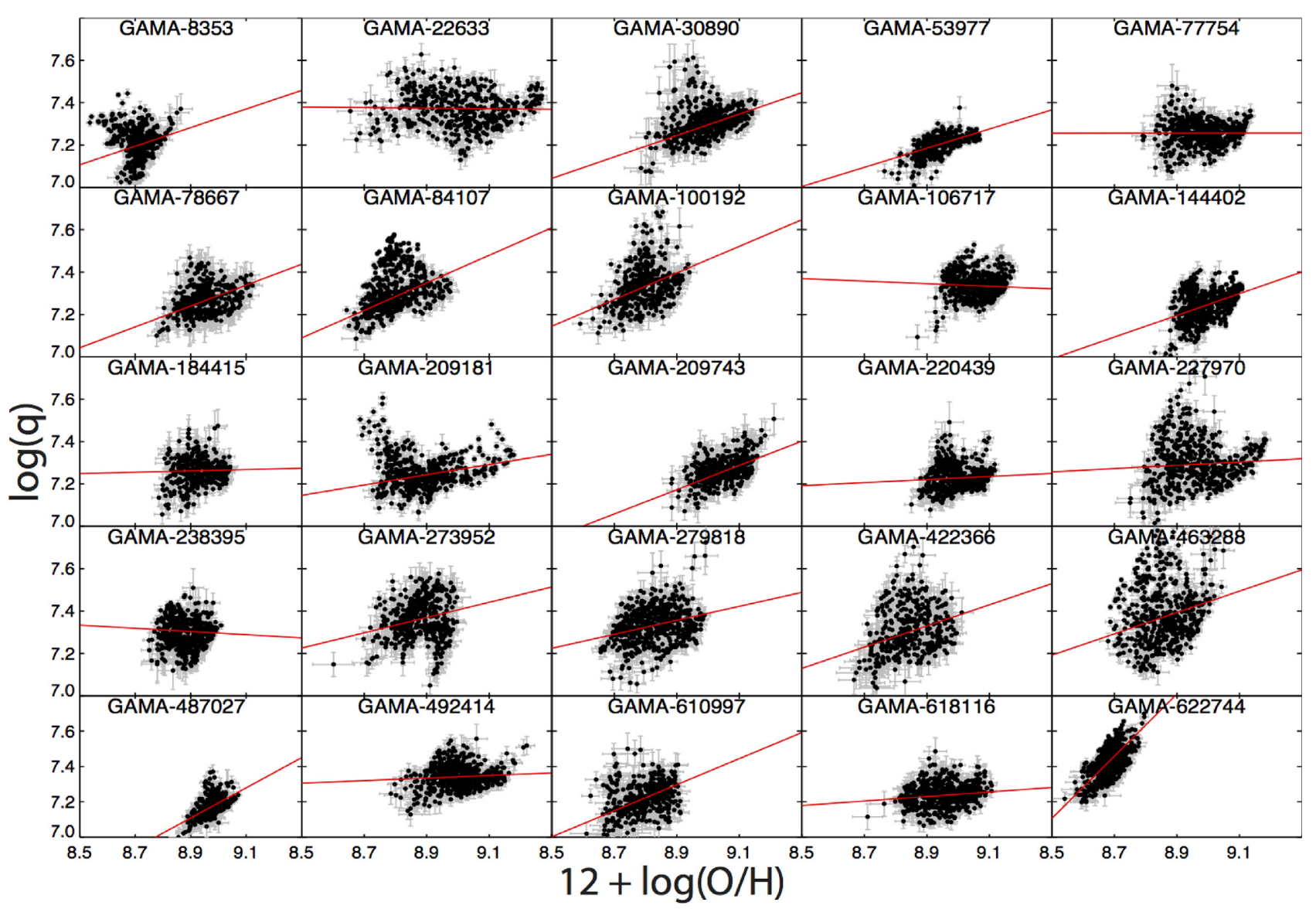

Figure 11. Relationship between the ionization parameter determined from the KK04 O32 diagnostic and the metallicity calculated from the KD02 N2O2 diagnostic. The best linear fit is given as a red line, and we summarize the results in Table C3.

\subsection{Ionization parameter effects on metallicity diagnostics}

In Fig. 8, we see that the typical ionization parameter range for our galaxy sample is $7.0<\log \left(q\left[\mathrm{~cm} \mathrm{~s}^{-1}\right]\right)<7.8$. An ionization parameter range this wide is enough to significantly affect the metallicity estimates for several metallicity diagnostics (Kewley \& Dopita 2002). As there are no discernible patterns in the distribution of ionization parameter, it makes it difficult to predict how the exclusion of ionization parameter will affect the metallicity distribution. We advise caution when interpreting results that have used metallicity diagnostics where ionization parameter has not been taken into account.

\section{SUMMARY}

We have presented metallicity and ionization parameter maps of 25 high-S/N face-on star-forming galaxies in DR1 of the SAMI Galaxy Survey. To account for their interdependence, metallicity and ionization parameter were determined simultaneously for individual spaxels using an iterative method involving the strong emission line diagnostics outlined in Kobulnicky \& Kewley (2004). We measure metallicity gradients as a function of galactocentric radius using robust line fitting routines. We find that the majority of galaxies exhibit a negative metallicity gradient with an average metallicity gradient of $-0.12 \pm 0.05 \mathrm{dex} / R_{\mathrm{e}}$ using the KK04 $\mathrm{R}_{23}$ diagnostic. Metallicity gradients show a weak negative correlation with the stellar mass of galaxies. Using the PP04 O3N2 metallicity diagnostic, we find an average metallicity gradient of $-0.10 \pm 0.06$ $\mathrm{dex} / R_{\mathrm{e}}$, which agrees with the gradients determined by Sánchez et al. (2012, 2014), Sánchez-Menguiano et al. (2016a), and Belfiore et al. (2017). Due to the unreliable $R_{25}$ measurements of the galaxies in our sample, we are unable to directly compare our metallicity gradient value to that in Ho et al. (2015). However, assuming $\mathrm{R}_{25}$ $=3.6 R_{\mathrm{e}}$ based on Williams et al. (2009), we find an average $\mathrm{N} 2 \mathrm{O} 2$ metallicity gradient of $-0.48 \pm 0.18$, consistent with that of Ho et al. (2015). Using the central metallicities of each galaxy based on the linear fits, we find that our galaxies are in agreement with the mass-metallicity relation polynomial presented in Kewley \& Ellison (2008) after applying a positive offset of $0.13 \mathrm{dex}$. The offset is likely a result of using interpolated central metallicities rather than the aperture average value as determined for SDSS. We show that the ionization parameter maps lack a significant or coherent structure unlike the metallicity maps. We do not see significant ionization parameter gradients like those presented in Kaplan et al. (2016); however, this could be due to sample selection differences or spatial resolution limitations. We do find a decrease in ionization parameter in the inter-arm regions of galaxies with resolvable spiral arms, indicating a possible correlation between the ionization parameter and SFR. However, for our galaxy sample, we find no significant correlations between the ionization parameter and SFR or sSFR. Until a better understanding is achieved on the distribution of ionization parameter, metallicity diagnostics must be used with care. We suggest that in order to obtain reliable metallicity maps, to either use a metallicity diagnostic that explicitly provides solutions for a range of ionization parameter like the one used in this study 
(e.g. KK04 R23), or use a metallicity diagnostic that is relatively invariant to changes in the ionization parameter (e.g. KD02 N2O2 or D16 N2S2).

\section{ACKNOWLEDGEMENTS}

The SAMI Galaxy Survey is based on observations made at the Anglo-Australian Telescope. The Sydney-AAO Multi-object Integral field spectrograph (SAMI) was developed jointly by the University of Sydney and the Australian Astronomical Observatory. The SAMI input catalogue is based on data taken from the Sloan Digital Sky Survey, the GAMA Survey, and the VST ATLAS Survey. The SAMI Galaxy Survey is funded by the Australian Research Council Centre of Excellence for All-sky Astrophysics (CAASTRO), through project number CE110001020, and other participating institutions. The SAMI Galaxy Survey website is http://sami-survey.org/.

Parts of this research were conducted by the Australian Research Council Centre of Excellence for All Sky Astrophysics in 3 Dimensions (ASTRO 3D), through project number CE170100013.

BG gratefully acknowledges the support of the Australian Research Council as the recipient of a Future Fellowship (FT140101202).

Support for AMM is provided by NASA through Hubble Fellowship grant \#HST-HF2-51377 awarded by the Space Telescope Science Institute, which is operated by the Association of Universities for Research in Astronomy, Inc., for NASA, under contract NAS5-26555.

JvdS is funded under Bland-Hawthorn's ARC Laureate Fellowship (FL140100278).

SFS thanks CONACYT programmes CB-285080 and DGAPAPAPIIT IA101217 grants for their support to this project.

SB acknowledges the funding support from the Australian Research Council through a Future Fellowship (FT140101166).

MSO acknowledges the funding support from the Australian Research Council through a Future Fellowship (FT140100255).

NS acknowledges support of a University of Sydney Postdoctoral Research Fellowship.

\section{REFERENCES}

Belfiore F. et al., 2017, MNRAS, 469, 151

Blanc G. A. et al., 2013, AJ, 145, 138

Bland-Hawthorn J., 2015, in Ziegler B. L., Combes F., Dannerbauer H., Verdugo M., eds, Proc. IAU Symp. 309, Galaxies in 3D across the Universe. Cambridge Univ. Press, Cambridge, p. 21

Bland-Hawthorn J. et al., 2011, Opt. Express, 19, 2649

Boissier S., Prantzos N., 1999, MNRAS, 307, 857

Bresolin F., Ryan-Weber E., Kennicutt R. C., Goddard Q., 2009, ApJ, 695, 580

Bresolin F., Kennicutt R. C., Ryan-Weber E., 2012, ApJ, 750, 122

Bryant J. J. et al., 2015, MNRAS, 447, 2857

Bundy K. et al., 2015, ApJ, 798, 7

Cappellari M. et al., 2011, MNRAS, 413, 813

Cappellari M. et al., 2013, MNRAS, 432, 1709

Cardelli J. A., Clayton G. C., Mathis J. S., 1989, ApJ, 345, 245

Charlot S., Longhetti M., 2001, MNRAS, 323, 887

Croom S. M. et al., 2012, MNRAS, 421, 872

Croom S. M., Allen J. T., Cortese L., Fogarty L., Ho I.-T., 2015, in Cappellari M., Courteau S., eds, Proc. IAU Symp. 311, Galaxy Masses as Constraints of Formation Models. Cambridge Univ. Press, Cambridge, p. 104

Davies R. L., Rich J. A., Kewley L. J., Dopita M. A., 2014, MNRAS, 439, 3835
Davies R. L. et al., 2016, MNRAS, 462, 1616

de Zeeuw P. T. et al., 2002, MNRAS, 329, 513

Dopita M. A., Evans I. N., 1986, ApJ, 307, 431

Dopita M. A. et al., 2006, ApJ, 647, 244

Dopita M. A., Rich J., Vogt F. P. A., Kewley L. J., Ho I.-T., Basurah H. M., Ali A., Amer M. A., 2014, Ap\&SS, 350, 741

Dopita M. A., Kewley L. J., Sutherland R. S., Nicholls D. C., 2016, Ap\&SS, 361,61

Driver S. P. et al., 2011, MNRAS, 413, 971

Drory N. et al., 2015, AJ, 149, 77

Edmunds M. G., Pagel B. E. J., 1984, MNRAS, 211, 507

Green A. W. et al., 2017, MNRAS, 475, 716

Groves B., Dopita M., Sutherland R., 2004, in Storchi-Bergmann T., Ho L. C., Schmitt H. R., eds, Proc. IAU Symp. 222, The Interplay Among Black Holes, Stars and ISM in Galactic Nuclei. Cambridge Univ. Press, Cambridge, p. 263

Heiderman A. L., Evans N. J., II, Gebhardt K., Blanc G., Davis T. A., Papovich C., Iono D., Yun M. S., 2011, in Salviander S., Green J. Pawlik A., eds, Proc. Frank N. Bash Symp. 2011: New Horizons in Astronomy. October 9-11, 2011, Austin, Texas, USA, p. 29. Available at: https://pos.sissa.it/cgi-bin/reader/conf.cgi?confid=149

Ho I.-T., Kudritzki R.-P., Kewley L. J., Zahid H. J., Dopita M. A., Bresolin F., Rupke D. S. N., 2015, MNRAS, 448, 2030

Ho I.-T. et al., 2016, Ap\&SS, 361, 280

Ho I.-T. et al., 2017, ApJ, 846, 39

Kaplan K. F. et al., 2016, MNRAS, 462, 1642

Karim A. et al., 2011, ApJ, 730, 61

Kelvin L. S. et al., 2012, MNRAS, 421, 1007

Kewley L. J., Dopita M. A., 2002, ApJS, 142, 35

Kewley L. J., Ellison S. L., 2008, ApJ, 681, 1183

Kewley L. J., Jansen R. A., Geller M. J., 2005, PASP, 117, 227

Kewley L. J., Groves B., Kauffmann G., Heckman T., 2006, MNRAS, 372, 961

Kewley L. J., Rupke D., Zahid H. J., Geller M. J., Barton E. J., 2010, ApJ, 721, L48

Kobulnicky H. A., Kewley L. J., 2004, ApJ, 617, 240

López-Sánchez Á. R., Mesa-Delgado A., López-Martín L., Esteban C., 2011, MNRAS, 411, 2076

López-Sánchez Á. R., Dopita M. A., Kewley L. J., Zahid H. J., Nicholls D. C., Scharwächter J., 2012, MNRAS, 426, 2630

López-Sánchez Á. R., Westmeier T., Esteban C., Koribalski B. S., 2015, MNRAS, 450, 3381

McCall M. L., Rybski P. M., Shields G. A., 1985, ApJS, 57, 1

McGaugh S. S., 1991, ApJ, 380, 140

Maiolino R. et al., 2008, A\&A, 488, 463

Makarov D., Prugniel P., Terekhova N., Courtois H., Vauglin I., 2014, A\&A, 570, A13

Marino R. A. et al., 2012, ApJ, 754, 61

Markwardt C. B., 2009, in Bohlender D. A., Durand D., Dowler P., eds, ASP Conf. Ser. Vol. 411, Astronomical Data Analysis Software and Systems XVIII. Astron. Soc. Pac., San Francisco, p. 251

Martin P., Roy J.-R., 1994, ApJ, 424, 599

Mast D. et al., 2014, A\&A, 561, A129

Matteucci F., Francois P., 1989, MNRAS, 239, 885

Medling A. M. et al., 2018, MNRAS, 475, 5194

Moustakas J., Kennicutt R. C., Jr., Tremonti C. A., Dale D. A., Smith J.-D. T., Calzetti D., 2010, ApJS, 190, 233

Osterbrock D. E., 1989, Astrophysics of Gaseous Nebulae and Active Galactic Nuclei. University Science Books, Mill Valley, CA

Owers M. S. et al., 2017, MNRAS, 468, 1824

Pagel B. E. J., Edmunds M. G., 1981, ARA\&A, 19, 77

Pagel B. E. J., Edmunds M. G., Blackwell D. E., Chun M. S., Smith G., 1979, MNRAS, 189, 95

Pagel B. E. J., Edmunds M. G., Smith G., 1980, MNRAS, 193, 219

Peng C. Y., Ho L. C., Impey C. D., Rix H.-W., 2002, AJ, 124, 266

Pettini M., Pagel B. E. J., 2004, MNRAS, 348, L59

Pilkington K. et al., 2012, A\&A, 540, A56

Pilyugin L. S., 2000, A\&A, 362, 325 
Rosales-Ortega F. F., Kennicutt R. C., Sánchez S. F., Díaz A. I., Pasquali A., Johnson B. D., Hao C. N., 2010, MNRAS, 405, 735

Rosales-Ortega F. F., Díaz A. I., Kennicutt R. C., Sánchez S. F., 2011, MNRAS, 415, 2439

Rupke D. S. N., Kewley L. J., Chien L.-H., 2010, ApJ, 723, 1255

Salpeter E. E., 1955, ApJ, 121, 161

Sánchez S. F. et al., 2012, A\&A, 546, A2

Sánchez S. F. et al., 2014, A\&A, 563, A49

Sánchez-Menguiano L. et al., 2016a, A\&A, 587, A70

Sánchez-Menguiano L. et al., 2016b, ApJ, 830, L40

Sánchez-Menguiano L. et al., 2018, A\&A, 609, A119

Schaefer A. L. et al., 2017, MNRAS, 464, 121

Taylor E. N. et al., 2011, MNRAS, 418, 1587

Torres-Peimbert S., Peimbert M., Fierro J., 1989, ApJ, 345, 186
Torrey P., Cox T. J., Kewley L., Hernquist L., 2012, ApJ, 746, 108

Tremonti C. A. et al., 2004, ApJ, 613, 898

van de Sande J. et al., 2017, ApJ, 835, 104

Vila-Costas M. B., Edmunds M. G., 1992, MNRAS, 259, 121

Vilchez J. M., Pagel B. E. J., Diaz A. I., Terlevich E., Edmunds M. G., 1988, MNRAS, 235, 633

Williams M. J., Bureau M., Cappellari M., 2009, MNRAS, 400, 1665

Yuan T.-T., Kewley L. J., Rich J., 2013, ApJ, 767, 106

Zaritsky D., Kennicutt R. C., Jr., Huchra J. P., 1994, ApJ, 420, 87

Zhang K. et al., 2017, MNRAS, 466, 3217

APPENDIX A: METALLICITY MAPS

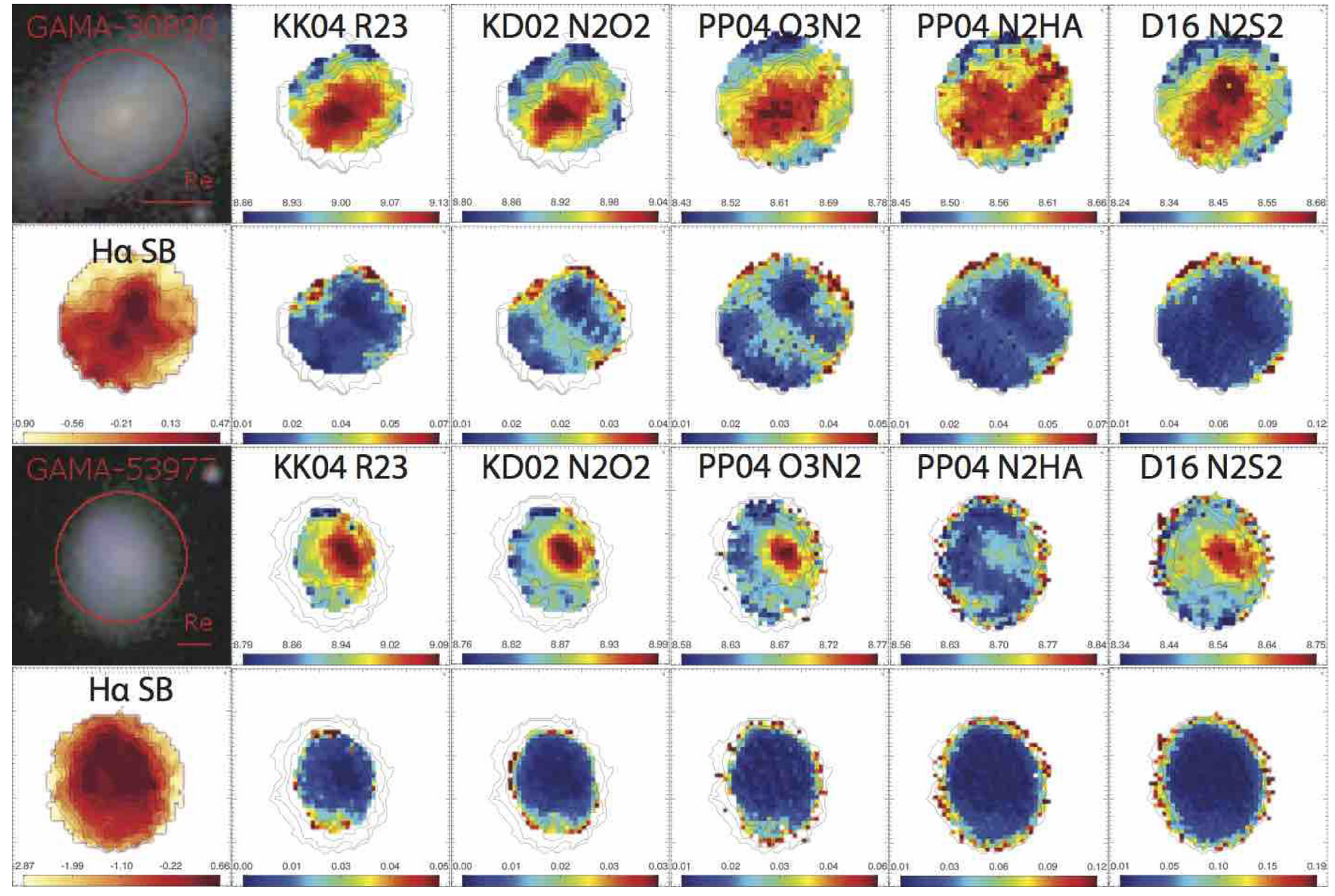

Figure A1. Same as Fig. 2 for GAMA-30890 and GAMA-53977. 


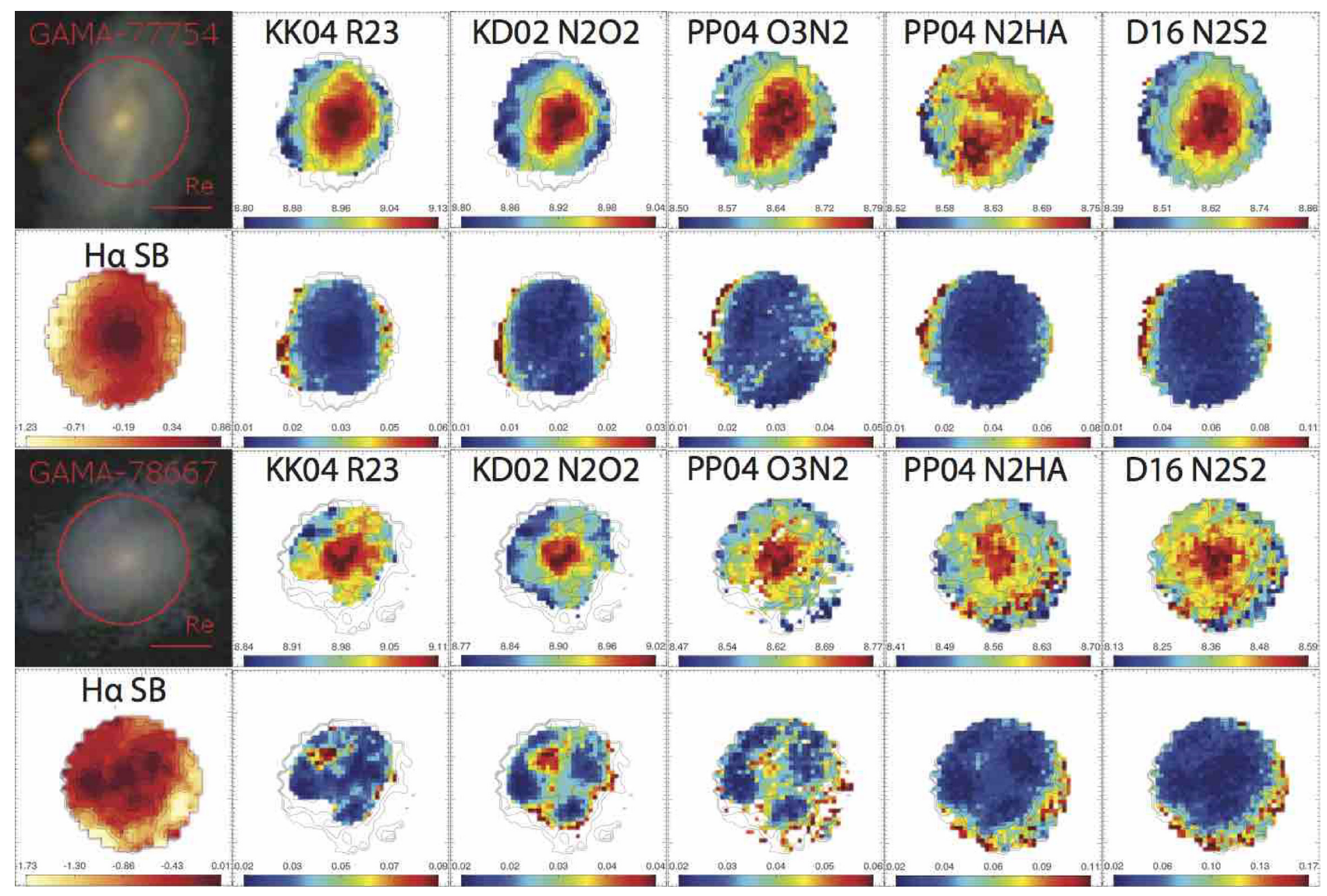

Figure A2. Same as Fig. 2 for GAMA-77754 and GAMA-78667. 


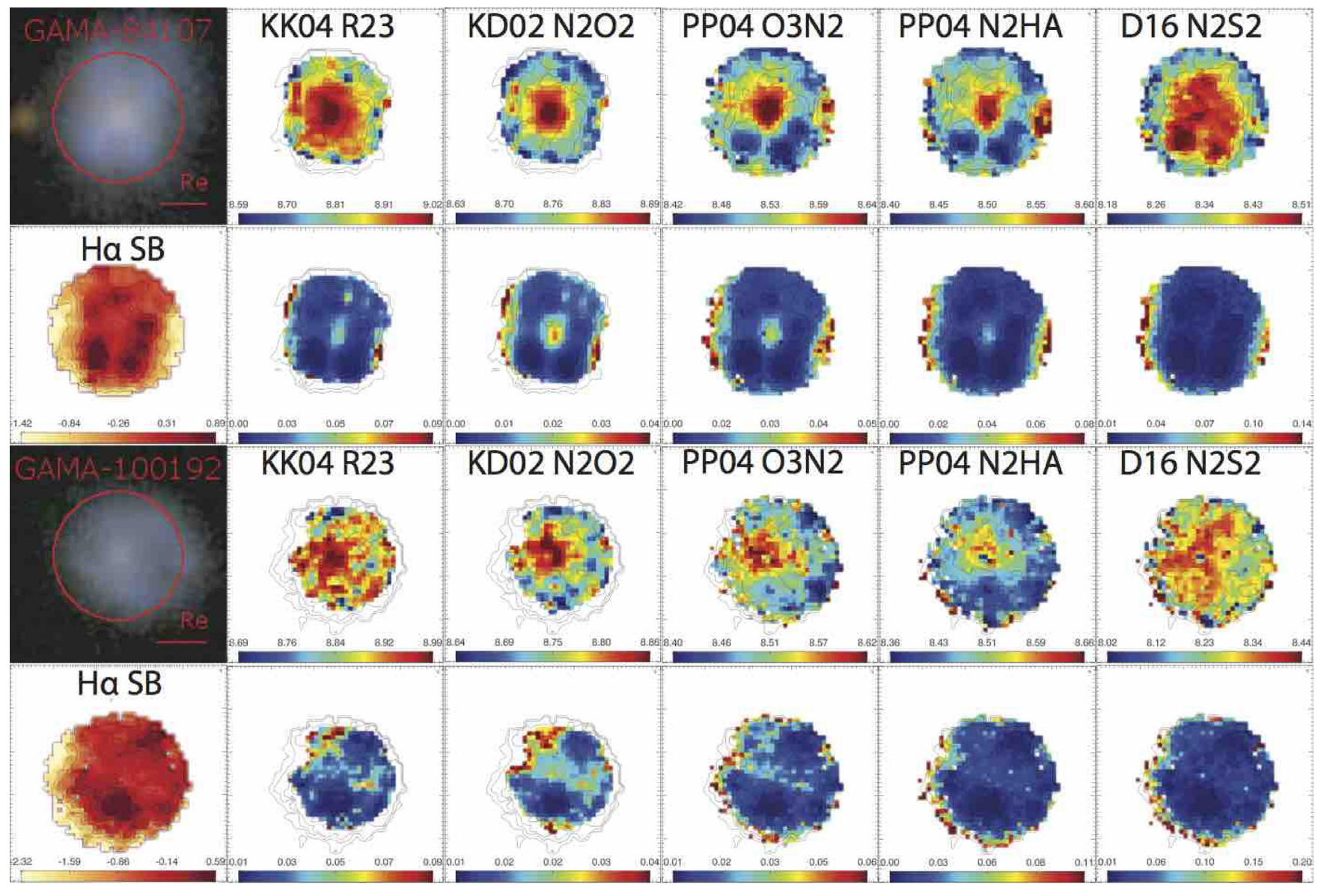

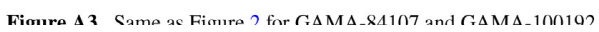

Figure A3. Same as Fig. 2 for GAMA-84107 and GAMA-100192. 


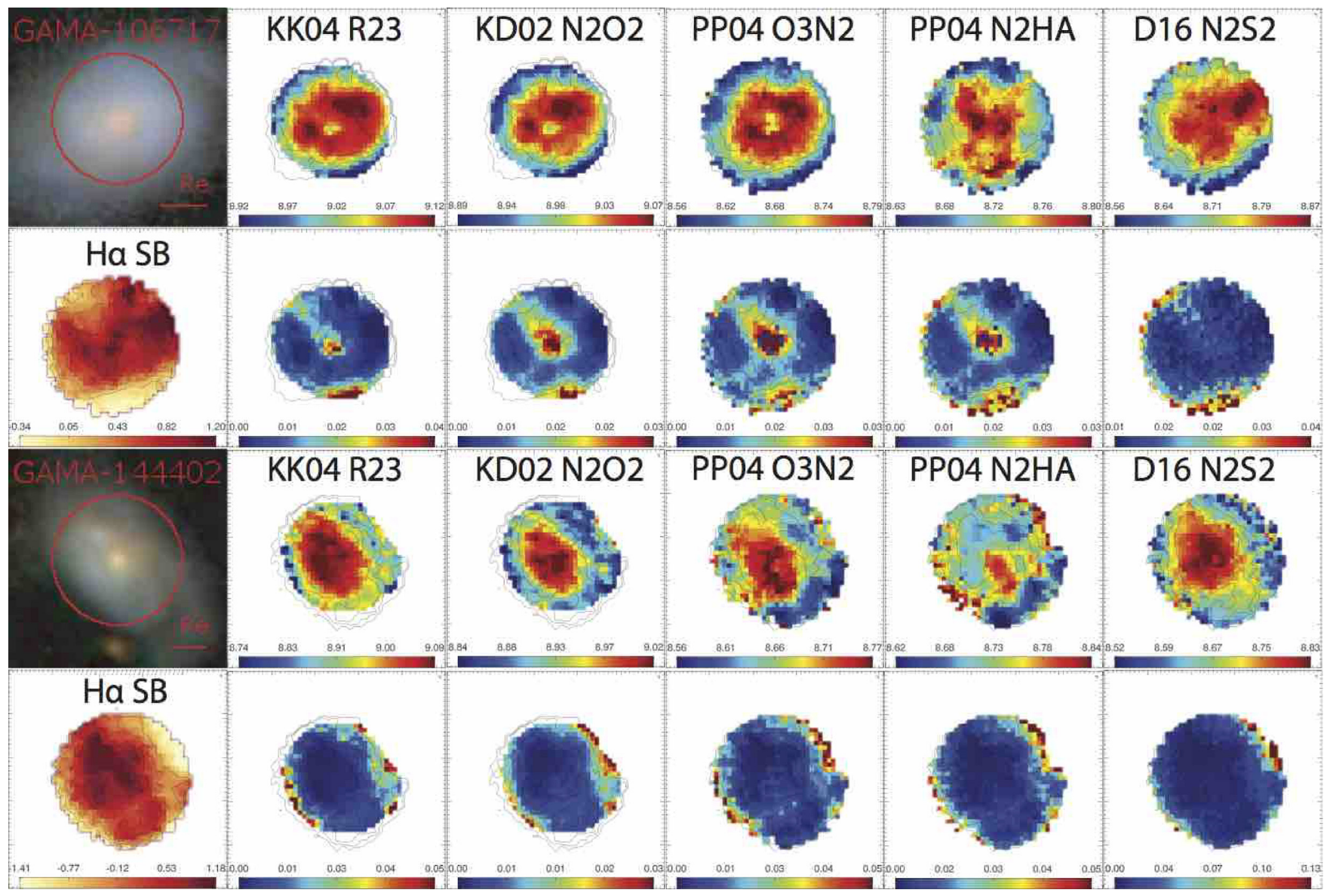

Figure A4. Same as Fig. 2 for GAMA-106717 and GAMA-144402. 


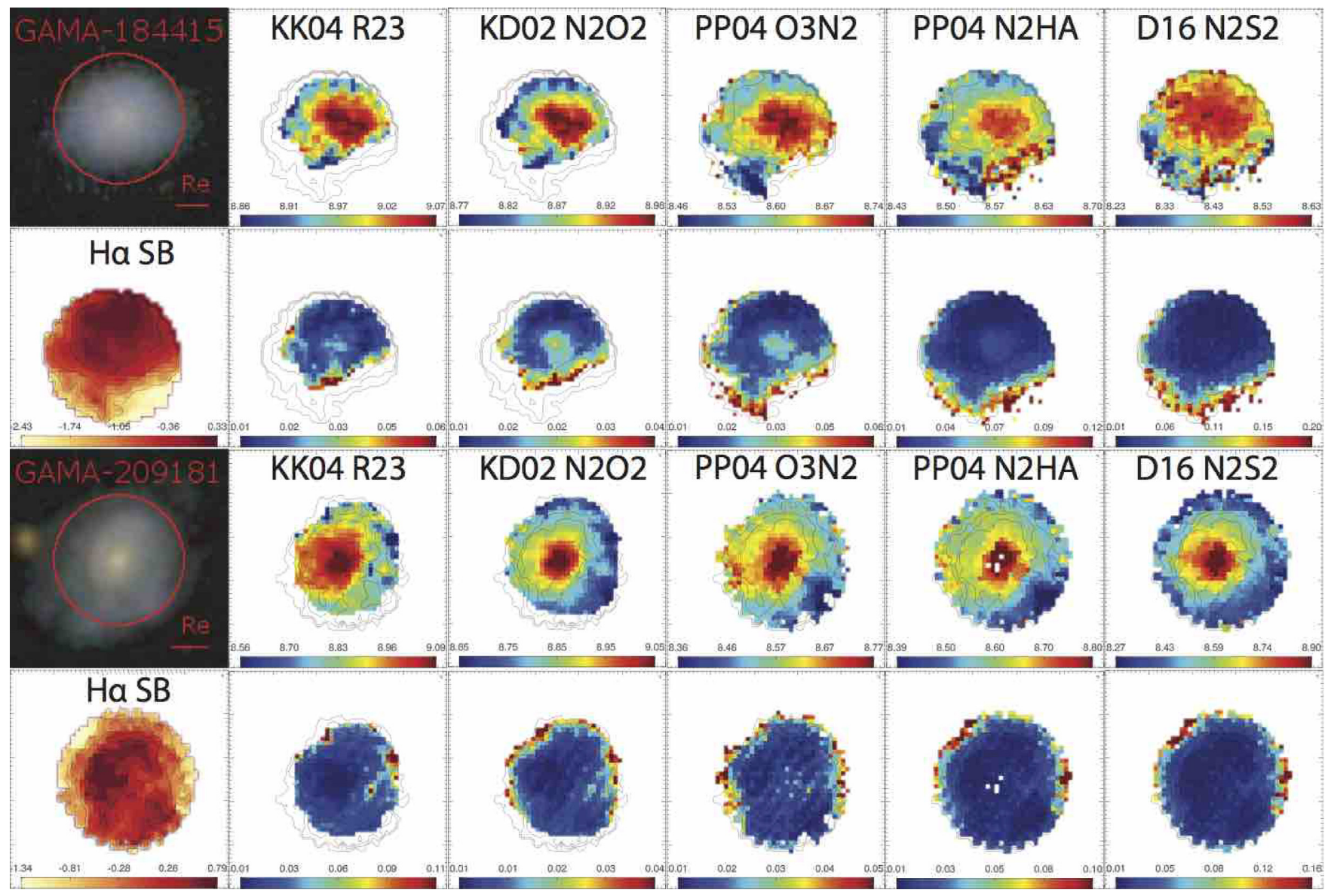

Figure A5. Same as Fig. 2 for GAMA-184415 and GAMA-209181. 


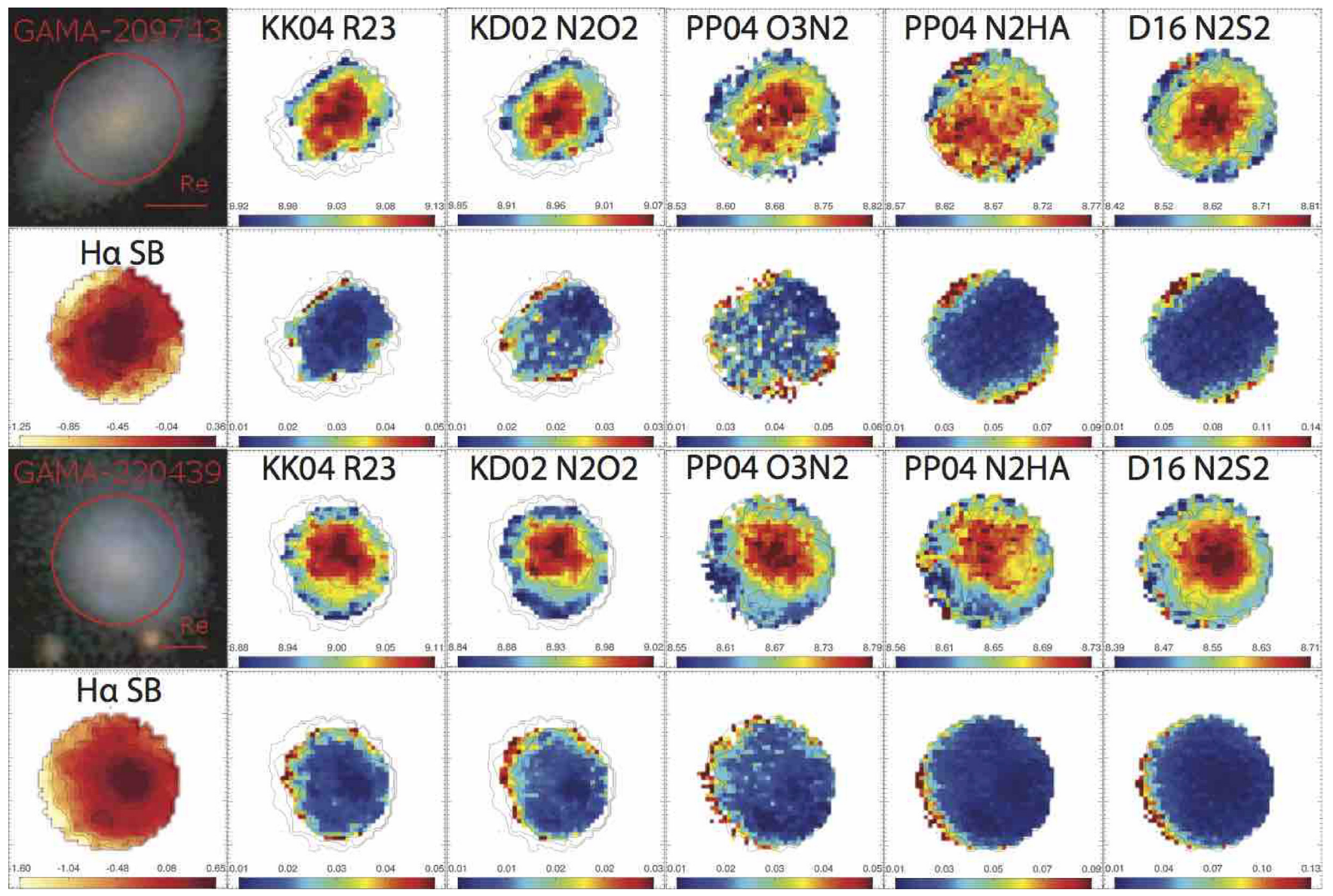

Figure A6. Same as Fig. 2 for GAMA-209743 and GAMA-220439. 


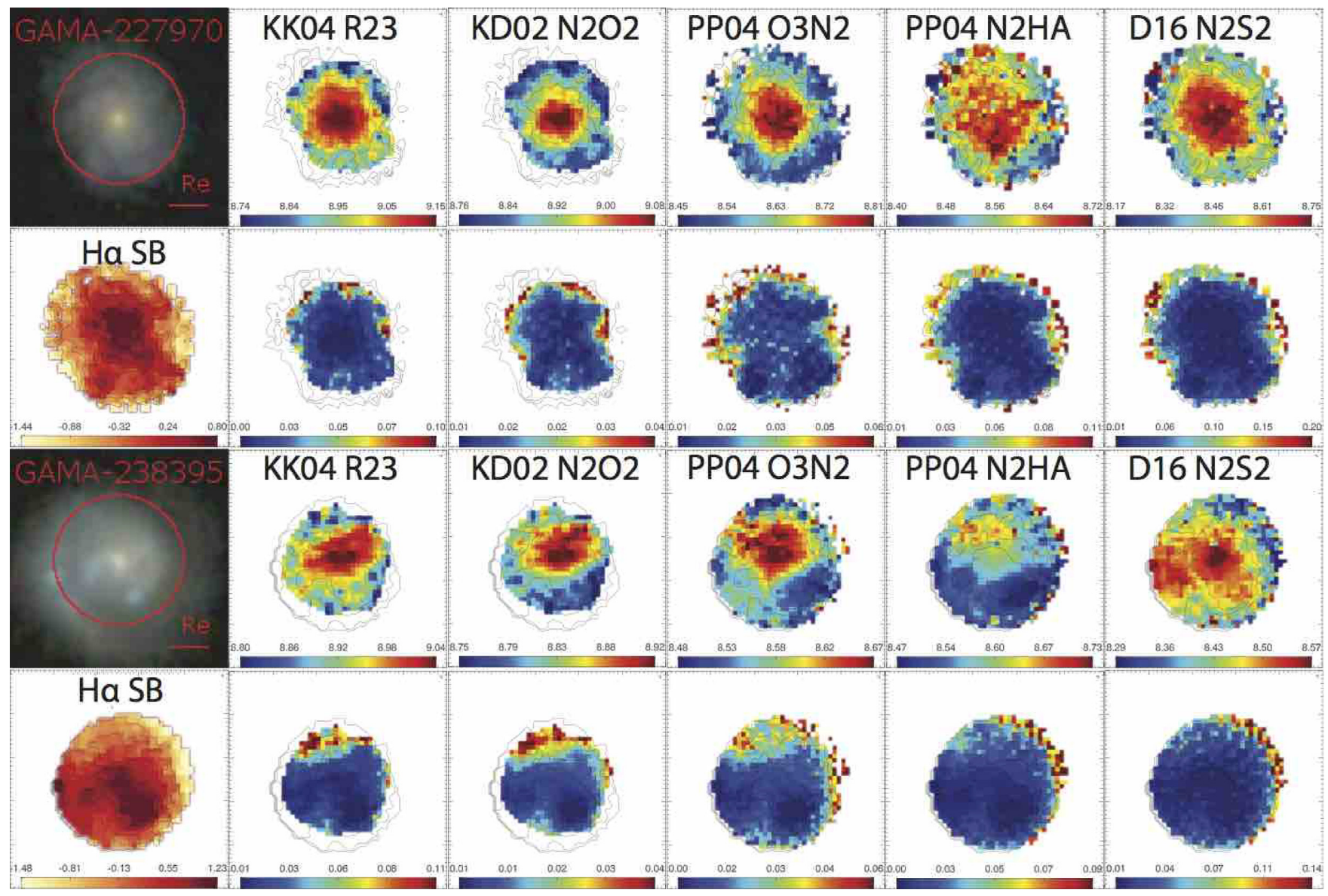

Figure A7. Same as Fig. 2 for GAMA-227970 and GAMA-238395. 


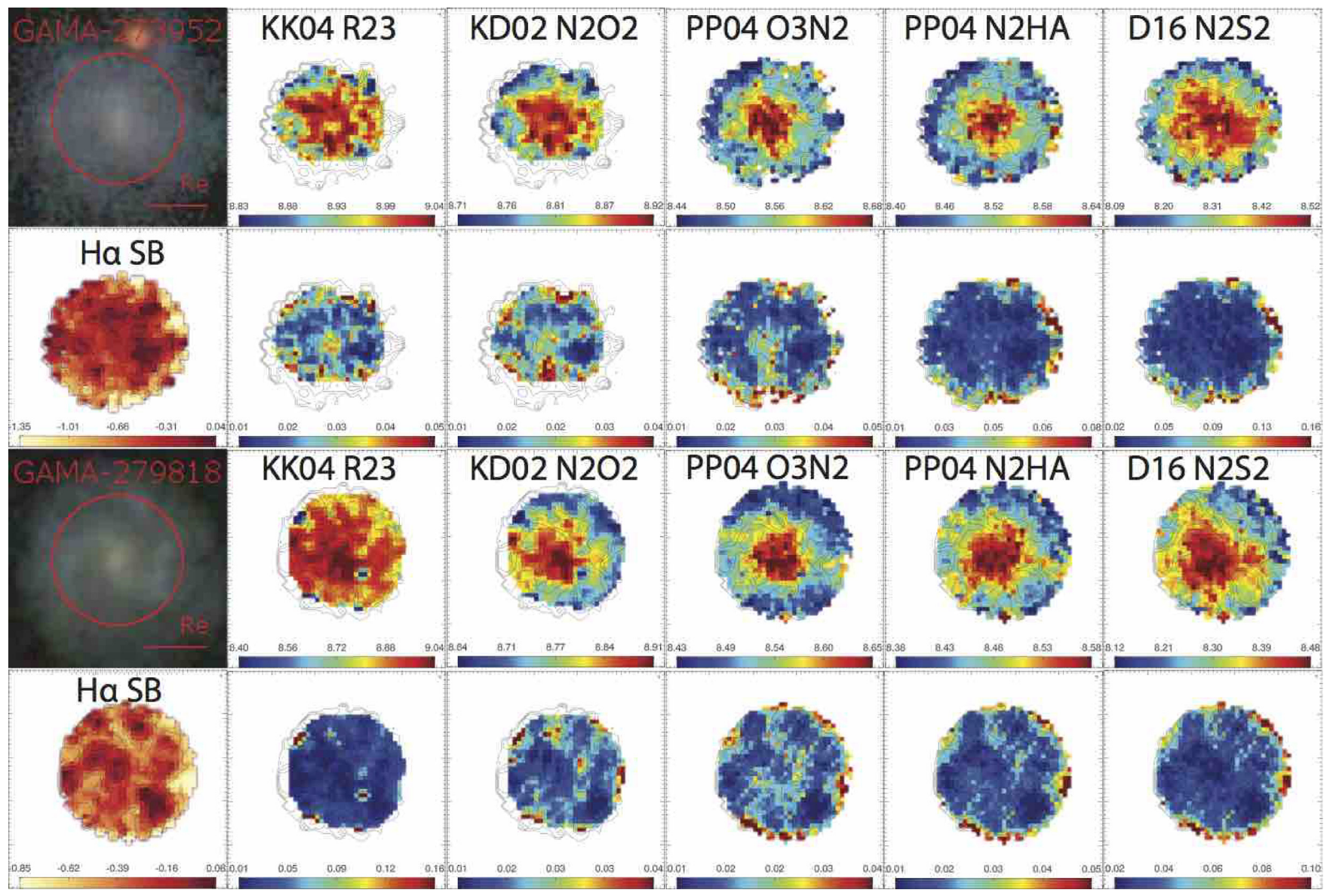

Figure A8. Same as Fig. 2 for GAMA-273952 and GAMA-279818. 


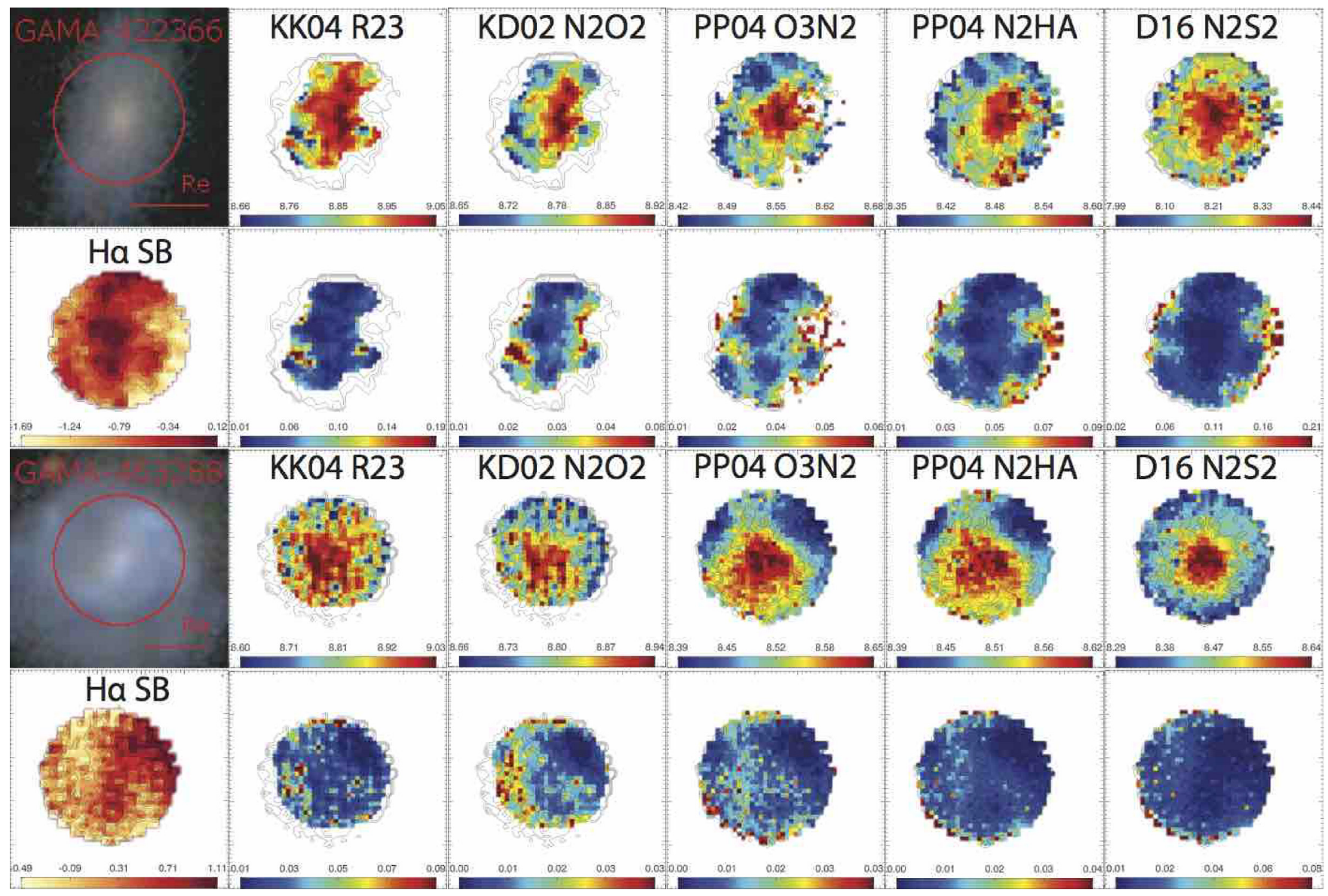

Figure A9. Same as Fig. 2 for GAMA-422366 and GAMA-463288. 


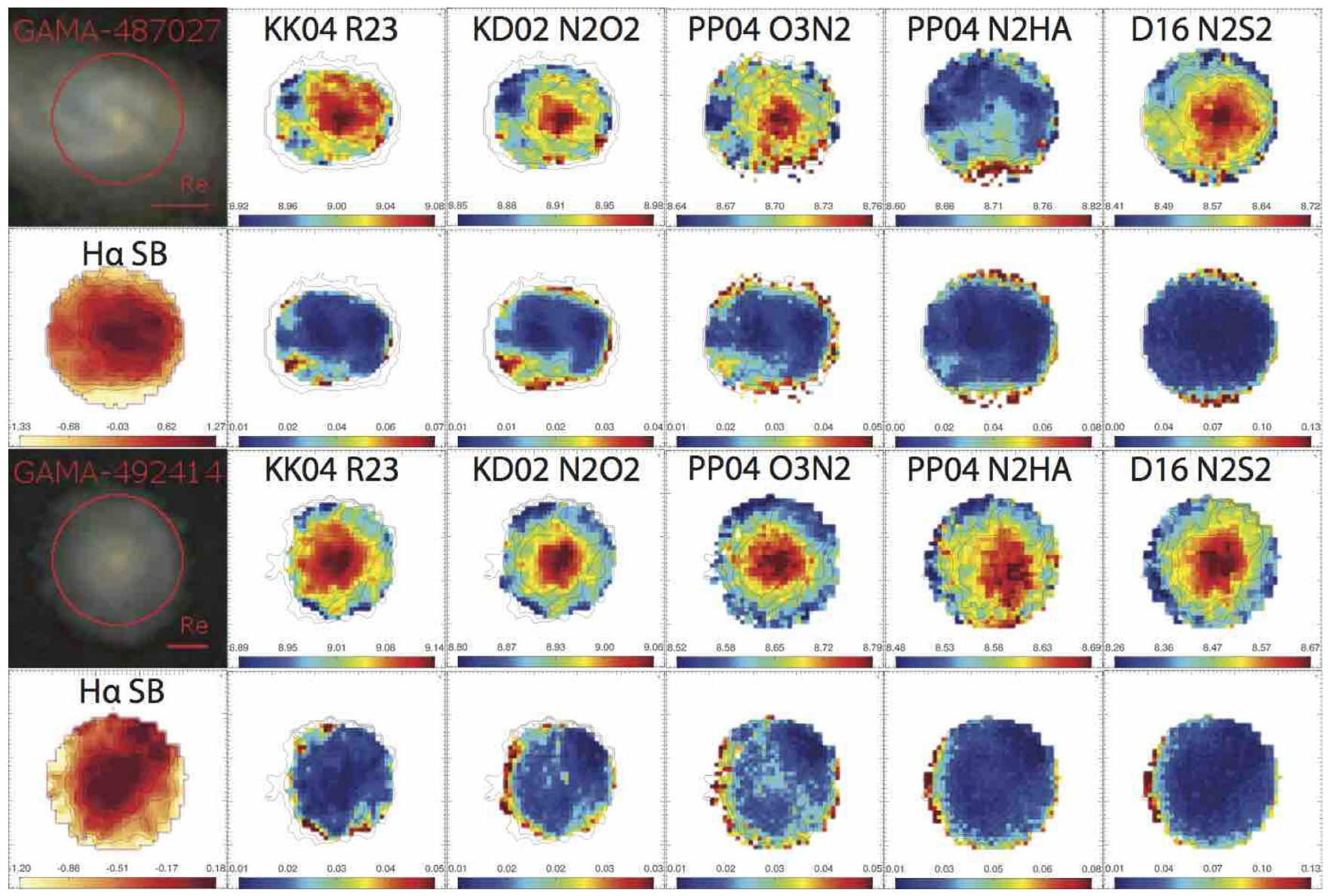

Figure A10. Same as Fig. 2 for GAMA-487027 and GAMA-492414. 


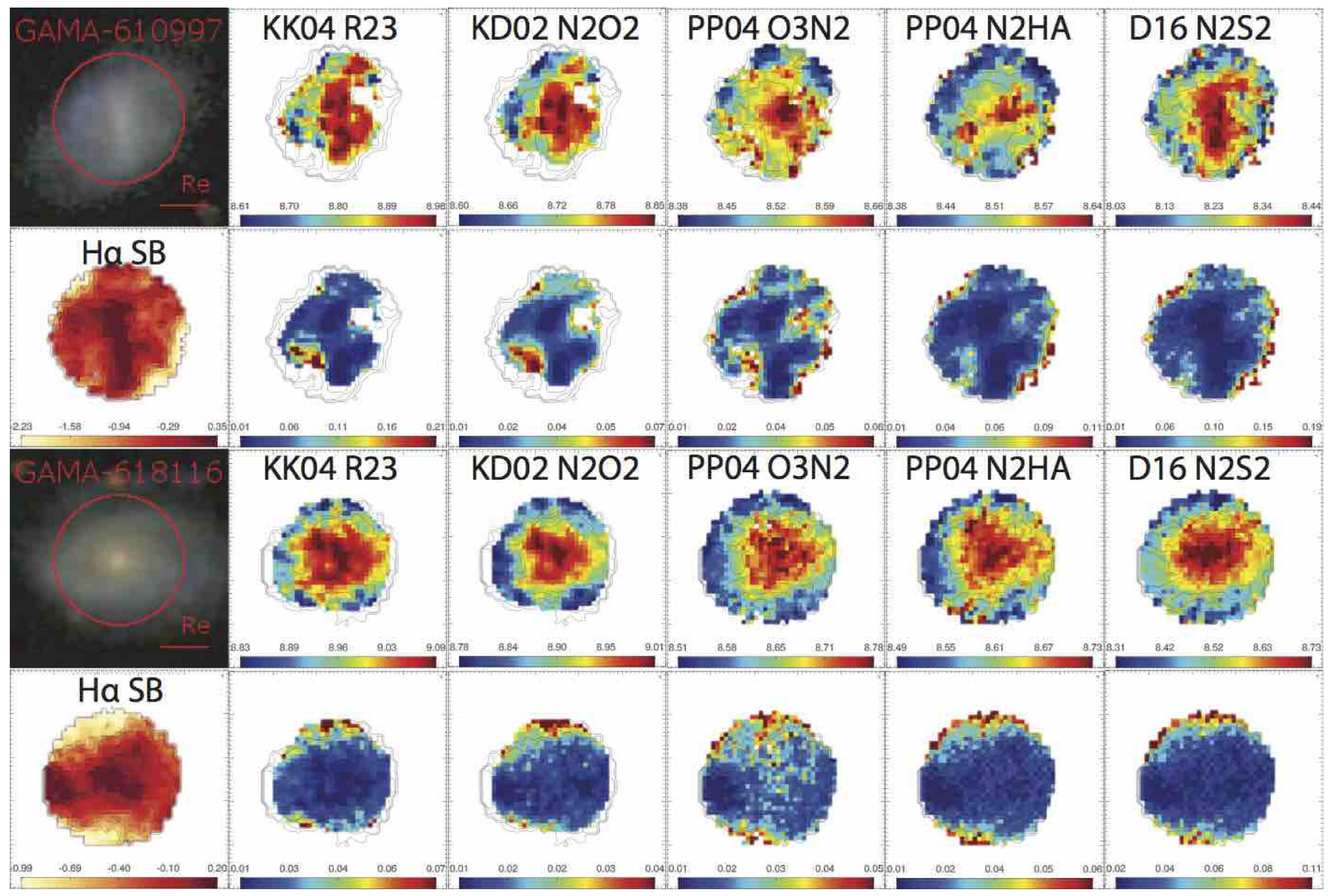

Figure A11. Same as Fig. 2 for GAMA-610997 and GAMA-618116. 
APPENDIX B: IONIZATION PARAMETER

MAPS

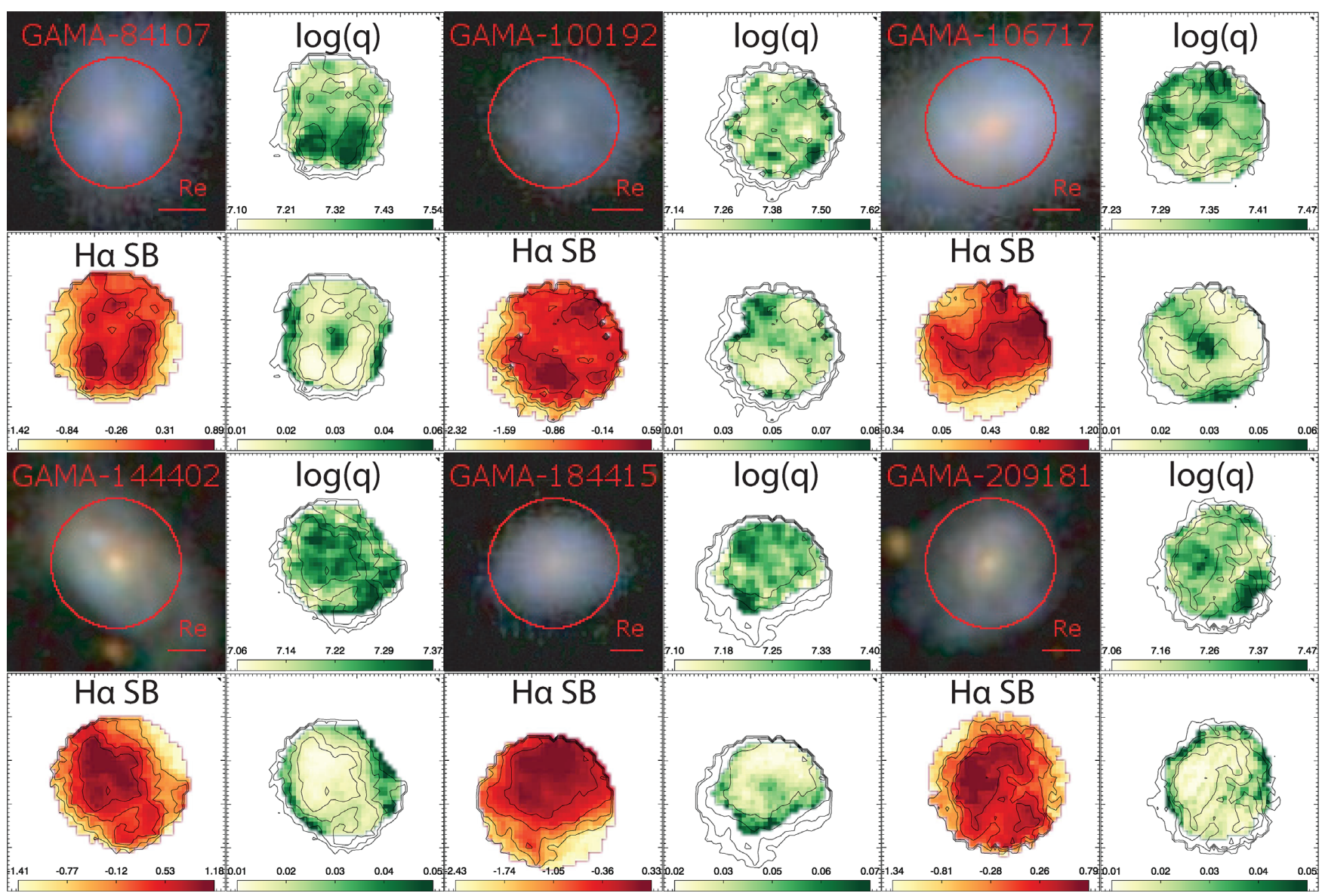

Figure B1. Sameas Fig. 8 for GAMA-84107, GAMA-100192, GAMA-106717, GAMA-144402, GAMA-184415, and GAMA-209181. 


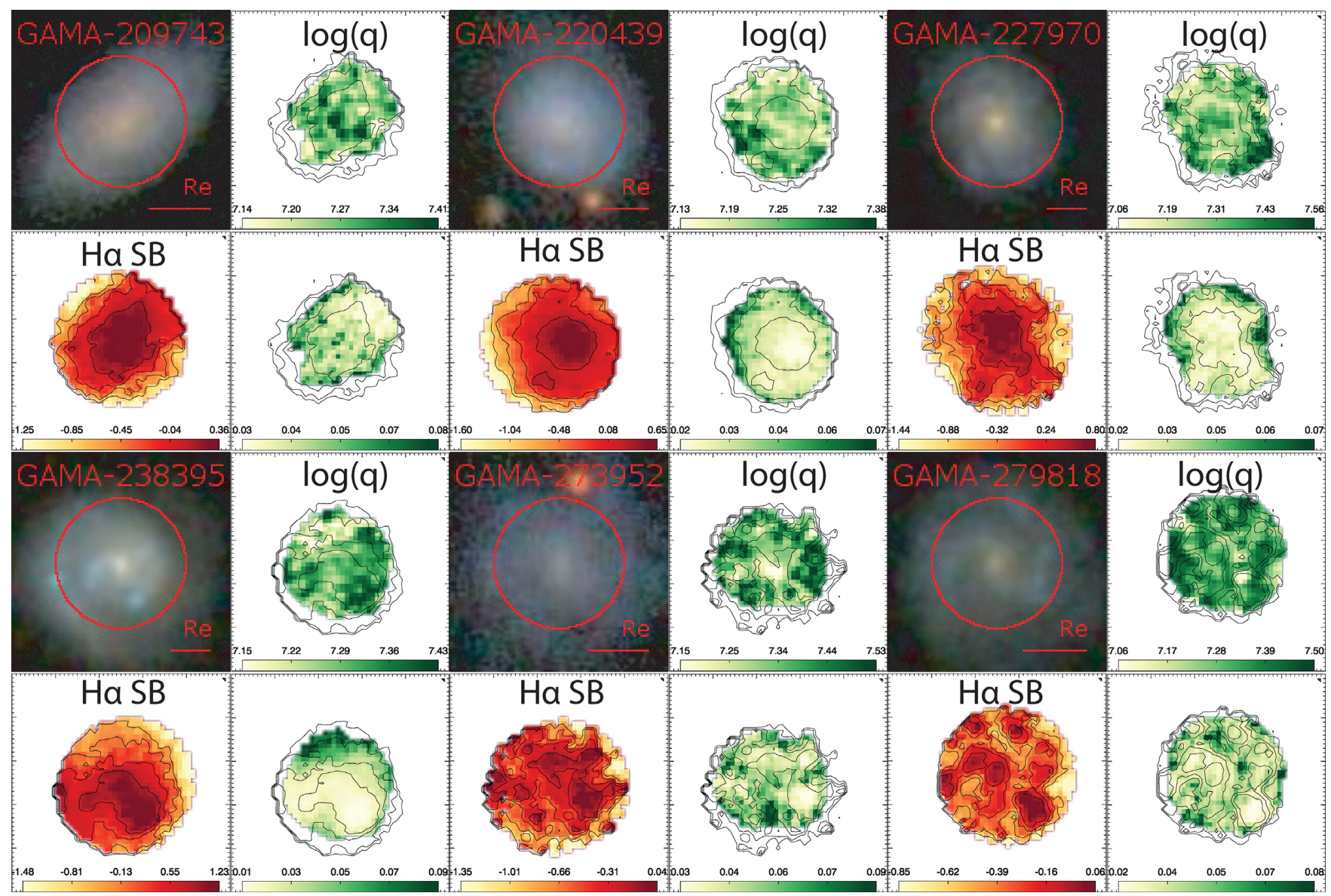

Figure B2. Same as Fig. 8 for GAMA-209743, GAMA-220439, GAMA-227970, GAMA-238395, GAMA-273952, and GAMA-279818. 


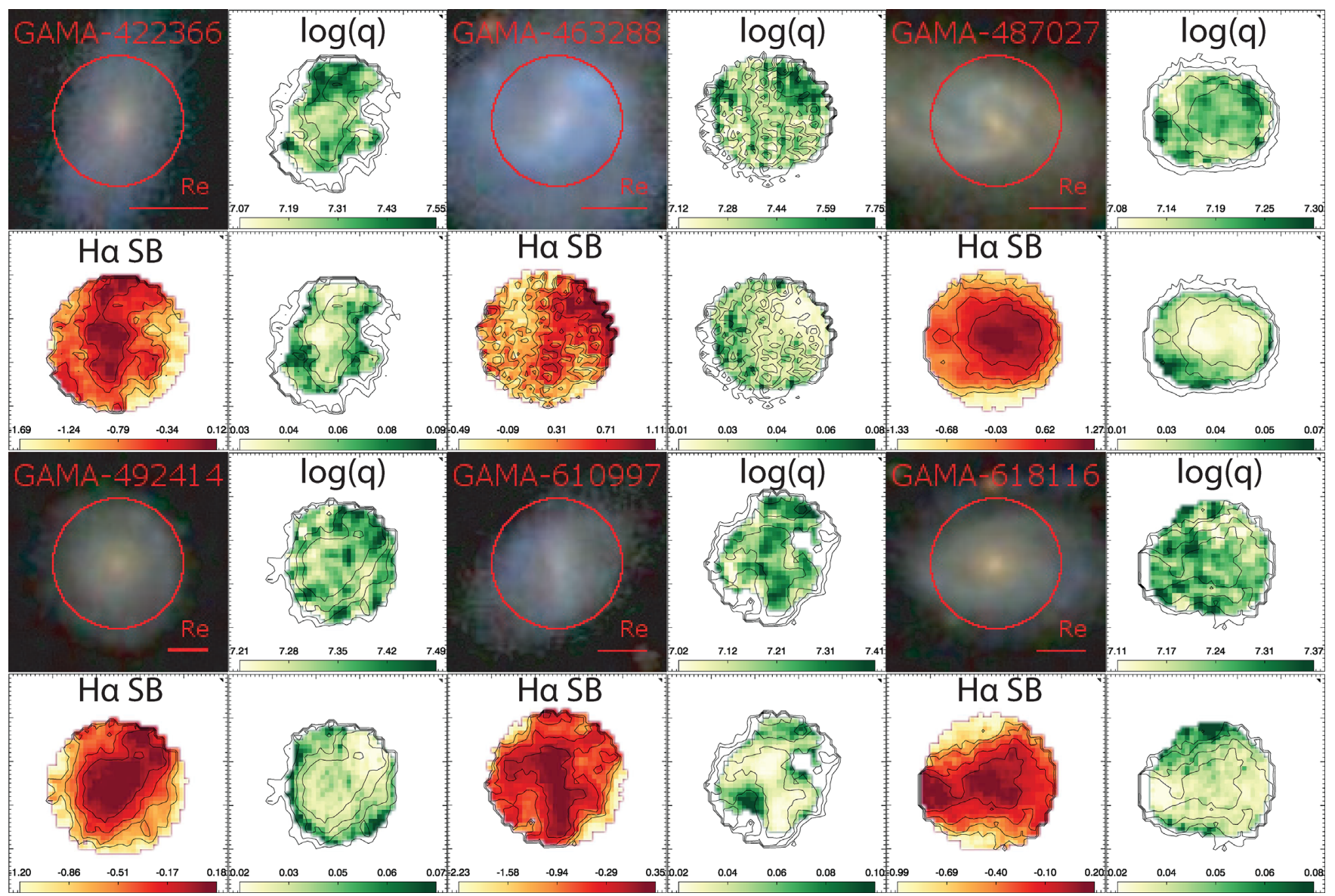

Figure B3. Same as Fig. 8 for GAMA-422366, GAMA-463288, GAMA-487027, GAMA-492414, GAMA-610997, and GAMA-618116. 


\section{APPENDIX C: TABLES}

Table C1. Linear fit parameters for Fig. 4.

\begin{tabular}{|c|c|c|c|c|}
\hline GAMA ID & $\begin{array}{c}\text { Intercept } \\
(12+\log (\mathrm{O} / \mathrm{H}))\end{array}$ & $\begin{array}{c}\text { Gradient } \\
(\operatorname{dex} / \log (\mathrm{SFR}))\end{array}$ & $\mathrm{rms}$ & PCC \\
\hline 008353 & $8.837 \pm 0.017$ & $0.037 \pm 0.010$ & 0.060 & 0.22 \\
\hline 022633 & $9.273 \pm 0.016$ & $0.148 \pm 0.010$ & 0.075 & 0.48 \\
\hline 030890 & $9.334 \pm 0.013$ & $0.146 \pm 0.007$ & 0.046 & 0.71 \\
\hline 053977 & $9.139 \pm 0.013$ & $0.091 \pm 0.008$ & 0.049 & 0.40 \\
\hline 077754 & $9.310 \pm 0.008$ & $0.182 \pm 0.005$ & 0.045 & 0.81 \\
\hline 078667 & $9.317 \pm 0.025$ & $0.132 \pm 0.012$ & 0.049 & 0.38 \\
\hline 084107 & $9.010 \pm 0.015$ & $0.074 \pm 0.008$ & 0.074 & 0.34 \\
\hline 100192 & $8.902 \pm 0.016$ & $-0.003 \pm 0.008$ & 0.063 & 0.02 \\
\hline 106717 & $9.214 \pm 0.006$ & $0.129 \pm 0.005$ & 0.032 & 0.63 \\
\hline 144402 & $9.149 \pm 0.007$ & $0.113 \pm 0.004$ & 0.046 & 0.71 \\
\hline 184415 & $9.089 \pm 0.019$ & $0.047 \pm 0.010$ & 0.050 & 0.34 \\
\hline 209181 & $9.167 \pm 0.021$ & $0.167 \pm 0.013$ & 0.101 & 0.49 \\
\hline 209743 & $9.493 \pm 0.014$ & $0.235 \pm 0.008$ & 0.034 & 0.75 \\
\hline 220439 & $9.296 \pm 0.011$ & $0.147 \pm 0.006$ & 0.037 & 0.65 \\
\hline 227970 & $9.301 \pm 0.014$ & $0.181 \pm 0.009$ & 0.077 & 0.63 \\
\hline 238395 & $8.992 \pm 0.008$ & $0.029 \pm 0.006$ & 0.052 & 0.36 \\
\hline 273952 & $8.951 \pm 0.023$ & $-0.010 \pm 0.010$ & 0.047 & -0.06 \\
\hline 279818 & $8.981 \pm 0.041$ & $0.027 \pm 0.018$ & 0.080 & 0.02 \\
\hline 422366 & $9.155 \pm 0.024$ & $0.082 \pm 0.011$ & 0.073 & 0.31 \\
\hline 463288 & $8.890 \pm 0.017$ & $0.002 \pm 0.010$ & 0.085 & -0.02 \\
\hline 487027 & $9.114 \pm 0.002$ & $0.066 \pm 0.002$ & 0.023 & 0.74 \\
\hline 492414 & $9.362 \pm 0.011$ & $0.146 \pm 0.006$ & 0.040 & 0.58 \\
\hline 610997 & $9.051 \pm 0.021$ & $0.076 \pm 0.010$ & 0.077 & 0.35 \\
\hline 618116 & $9.461 \pm 0.019$ & $0.221 \pm 0.009$ & 0.049 & 0.46 \\
\hline 622744 & $8.879 \pm 0.006$ & $0.024 \pm 0.004$ & 0.042 & 0.28 \\
\hline
\end{tabular}

Table C2. Linear fit parameters for Fig. 10.

\begin{tabular}{lcrrr}
\hline GAMA ID & $\begin{array}{c}\text { Intercept } \\
(\log (q))\end{array}$ & \multicolumn{1}{c}{$\begin{array}{c}\text { Gradient } \\
(\text { dex } / \log (\mathrm{SFR}))\end{array}$} & $\mathrm{rms}$ & \multicolumn{1}{c}{ PCC } \\
\hline 008353 & $7.570 \pm 0.019$ & $0.213 \pm 0.011$ & 0.065 & 0.58 \\
022633 & $7.457 \pm 0.016$ & $0.053 \pm 0.010$ & 0.076 & 0.15 \\
030890 & $7.368 \pm 0.018$ & $0.036 \pm 0.010$ & 0.059 & -0.02 \\
053977 & $7.289 \pm 0.011$ & $0.052 \pm 0.007$ & 0.040 & 0.28 \\
077754 & $7.242 \pm 0.010$ & $-0.009 \pm 0.006$ & 0.051 & -0.08 \\
078667 & $7.103 \pm 0.034$ & $-0.075 \pm 0.015$ & 0.062 & -0.21 \\
084107 & $7.602 \pm 0.018$ & $0.166 \pm 0.010$ & 0.084 & 0.53 \\
100192 & $7.444 \pm 0.028$ & $0.059 \pm 0.014$ & 0.083 & 0.20 \\
106717 & $7.364 \pm 0.008$ & $0.025 \pm 0.007$ & 0.047 & 0.21 \\
144402 & $7.323 \pm 0.009$ & $0.051 \pm 0.006$ & 0.061 & 0.33 \\
184415 & $7.396 \pm 0.023$ & $0.071 \pm 0.012$ & 0.054 & 0.02 \\
209181 & $7.367 \pm 0.016$ & $0.074 \pm 0.009$ & 0.069 & 0.32 \\
209743 & $7.341 \pm 0.027$ & $0.044 \pm 0.015$ & 0.057 & 0.14 \\
220439 & $7.174 \pm 0.015$ & $-0.032 \pm 0.008$ & 0.053 & -0.13 \\
227970 & $7.221 \pm 0.021$ & $-0.045 \pm 0.013$ & 0.096 & -0.12 \\
238395 & $7.388 \pm 0.009$ & $0.063 \pm 0.007$ & 0.060 & 0.38 \\
273952 & $7.363 \pm 0.047$ & $0.001 \pm 0.021$ & 0.088 & -0.03 \\
279818 & $7.437 \pm 0.041$ & $0.046 \pm 0.019$ & 0.074 & -0.01 \\
422366 & $7.376 \pm 0.049$ & $0.029 \pm 0.022$ & 0.107 & 0.10 \\
463288 & $7.573 \pm 0.029$ & $0.121 \pm 0.017$ & 0.134 & 0.28 \\
487027 & $7.238 \pm 0.006$ & $0.046 \pm 0.005$ & 0.043 & 0.23 \\
492414 & $7.368 \pm 0.020$ & $0.012 \pm 0.010$ & 0.062 & 0.05 \\
610997 & $7.358 \pm 0.027$ & $0.069 \pm 0.013$ & 0.084 & 0.12 \\
618116 & $7.182 \pm 0.020$ & $-0.028 \pm 0.010$ & 0.055 & -0.16 \\
622744 & $7.677 \pm 0.011$ & $0.144 \pm 0.006$ & 0.060 & 0.65 \\
\hline & & & &
\end{tabular}

Table C3. Linear fit parameters for Fig. 11.

\begin{tabular}{lrrrr}
\hline GAMA ID & \multicolumn{1}{c}{$\begin{array}{c}\text { Intercept } \\
(\log (q))\end{array}$} & $\begin{array}{l}\text { Gradient } \\
((\mathrm{dex} / \mathrm{Z}))\end{array}$ & $\mathrm{rms}$ & PCC \\
\hline 008353 & $2.843 \pm 0.644$ & $0.502 \pm 0.074$ & 0.083 & 0.28 \\
022633 & $7.517 \pm 0.291$ & $-0.016 \pm 0.033$ & 0.079 & 0.01 \\
030890 & $2.132 \pm 0.333$ & $0.578 \pm 0.037$ & 0.051 & 0.31 \\
053977 & $2.612 \pm 0.314$ & $0.517 \pm 0.035$ & 0.036 & 0.67 \\
077754 & $7.250 \pm 0.298$ & $0.001 \pm 0.033$ & 0.052 & 0.02 \\
078667 & $2.256 \pm 0.436$ & $0.563 \pm 0.049$ & 0.054 & 0.33 \\
084107 & $0.777 \pm 0.438$ & $0.743 \pm 0.050$ & 0.066 & 0.41 \\
100192 & $1.045 \pm 0.630$ & $0.718 \pm 0.072$ & 0.079 & 0.38 \\
106717 & $7.953 \pm 0.381$ & $-0.069 \pm 0.042$ & 0.049 & 0.11 \\
144402 & $2.011 \pm 0.495$ & $0.586 \pm 0.055$ & 0.065 & 0.42 \\
184415 & $6.944 \pm 0.508$ & $0.036 \pm 0.057$ & 0.058 & 0.13 \\
209181 & $4.796 \pm 0.308$ & $0.277 \pm 0.035$ & 0.070 & 0.15 \\
209743 & $1.378 \pm 0.455$ & $0.655 \pm 0.051$ & 0.052 & 0.51 \\
220439 & $6.491 \pm 0.389$ & $0.082 \pm 0.044$ & 0.051 & 0.15 \\
227970 & $6.496 \pm 0.472$ & $0.090 \pm 0.053$ & 0.097 & 0.12 \\
238395 & $8.063 \pm 0.503$ & $-0.086 \pm 0.057$ & 0.065 & 0.02 \\
273952 & $3.725 \pm 0.630$ & $0.412 \pm 0.071$ & 0.085 & 0.16 \\
279818 & $4.015 \pm 0.424$ & $0.378 \pm 0.048$ & 0.078 & 0.44 \\
422366 & $2.275 \pm 0.684$ & $0.571 \pm 0.078$ & 0.102 & 0.33 \\
463288 & $2.297 \pm 0.705$ & $0.576 \pm 0.080$ & 0.131 & 0.30 \\
487027 & $-1.538 \pm 0.421$ & $0.977 \pm 0.047$ & 0.034 & 0.56 \\
492414 & $6.602 \pm 0.382$ & $0.083 \pm 0.043$ & 0.062 & 0.17 \\
610997 & $-0.171 \pm 0.462$ & $0.844 \pm 0.053$ & 0.071 & 0.40 \\
618116 & $5.933 \pm 0.305$ & $0.147 \pm 0.034$ & 0.055 & 0.19 \\
622744 & $-9.987 \pm 0.721$ & $2.011 \pm 0.083$ & 0.068 & 0.73 \\
\hline & & & &
\end{tabular}

This paper has been typeset from a $\mathrm{T}_{\mathrm{E}} \mathrm{X} / \mathrm{L} \mathrm{T}_{\mathrm{E}} \mathrm{X}$ file prepared by the author. 